\title{
BIOSTRATIGRAPHIE DES MIOSPORES ET DES CHITINOZOAIRES DU SILURIEN SUPÉRIEUR ET DU DÉVONIEN DANS LE BASSIN D'ILLIZI
}

\author{
(S.E. DU SAHARA ALGÉRIEN)
}

\author{
par \\ KHEIRA BOUMENDJEL*, STANISLAS LOBOZIAK**, FLORENTIN PARIS***, PHILIPPE STEEMANS**** \\ \& MAURICE STREEL***
}

\section{RÉSUMÉ}

Des miospores et des Chitinozoaires bien conservés ont été extraits de carottes de sondage provenant du bassin d'Tllizi. L'analyse détaillée de la distribution verticale de ces microfossiles permet de dater avec précision les diverses formations du Silurien supérieur et du Dévonien, reconnues par sondage dans le SudEst du Sahara algérien. Cette étude biostratigraphique démontre d'autre part l'existence d'importantes lacunes liées à des émersions périodiques.

\begin{abstract}
.
Core samples from the Illizi basin yielded well preserved miospores and Chitinozoa. The detailed study of the range of these microfossils allows accurate age assignment for upper Silurian and Devonian subsurface strata of the southeastern part of the algerian Sahara. On the other hand, these biostratigraphical data demonstrate the occurrence of important stratigraphical gaps related to recurrent emersions.
\end{abstract}

MOTS-CLÉS : MIOSPORES, CHITINOZOAIRES, BIOSTRATIGRAPHIE, SILURIEN, DÉVONIEN, SAHARA.

KEY-WORDS : MIOSPORES, CHITINOZOA, BIOSTRATIGRAPHY, SILURIAN, DEVONIAN, SAHARA.

* Laboratoire de Paléontologie et Stratigraphie, Université de Rennes I; adresse actuelle : Laboratoire de Paléontologie, D.L.C.H., SONATRACH, Boumerdes, Algérie.

** Laboratoire de Paléobotanique et Biostratigraphie du Paléozoïque (GRECO 7 du CNRS), Université des Sciences et Techniques de Lille, 59655 Villeneuve d'Ascq Cedex, France.

*** Laboratoire de Paléontologie et Stratigraphie (GRECO 7 du CNRS), Université de Rennes I, 35042 Rennes Cedex, France.

**** Laboratoire de Paléobotanique et Paléopalynologie, Université de Liège, 7 place du XX Août, B 4000 Liège, Belgique. 


\section{INTRODUCTION}

Dans le Sud-Est du Sahara algérien, les terrains du Paléozoïque inférieur; exposés dans les Tassili N'Ajjer, s'ennoyent vers le Nord sous le Carbonifère puis sous la couverture mésozoïque du Bassin d'Illizi. Cette vaste structure communique vers le Nord-Est avec le bassin de Rhadames dont elle est séparee vers le SudEst par le môle de Tihemboka. Ce bassin d'Illizi est d'autre part limité vers l'Ouest par le haut fond d'Amguid-El Biod et, vers le Nord, par la Hamada du Tinrhert (fig. 1). Les séquences cambriennes à carbonifères représentées dans ce bassin ont été largement explorées par de nombreux sondages pétroliers. La succession lithostratigraphique, ainsi que les variations latérales de faciès et de puissance, y sont donc bien connues, en particulier dans le Silurien supérieur et le Dévonien (Jardiné \& Yapaudjian 1968). La datation précise de ces terrains et notamment l'évaluation de l'importance de certaines lacunes se sont en revanche souvent heurtées à la rareté de macrofossiles exploitables stratigraphiquement. Les résultats les plus probants ont été obtenus grâce aux microfossiles organiques, en particulier les Acritarches qui ont fait l'objet de deux publications synthétiques (Jardiné \& Yapaudjian 1968; Jardiné et alii 1974).

Dans la présente étude, nous nous proposons de préciser les attributions stratigraphiques antérieures en confrontant les données des Chitinozoaires et des miospores. Nous avons préféré concentrer nos investigations sur un puits assez bien carotté (TRN 3), plutôt que d'intégrer les données provenant de multiples sondages mais n'offrant aucune certitude quant à la position relative des prélèvements pris en compte. Nous pensons ainsi disposer d'un contrôle plus rigoureux pour établir la distribution verticale des taxons. Un second puits (EAL 1) a eté testé pour vérifier le caractère régional de la distribution des Chitinozoaires.

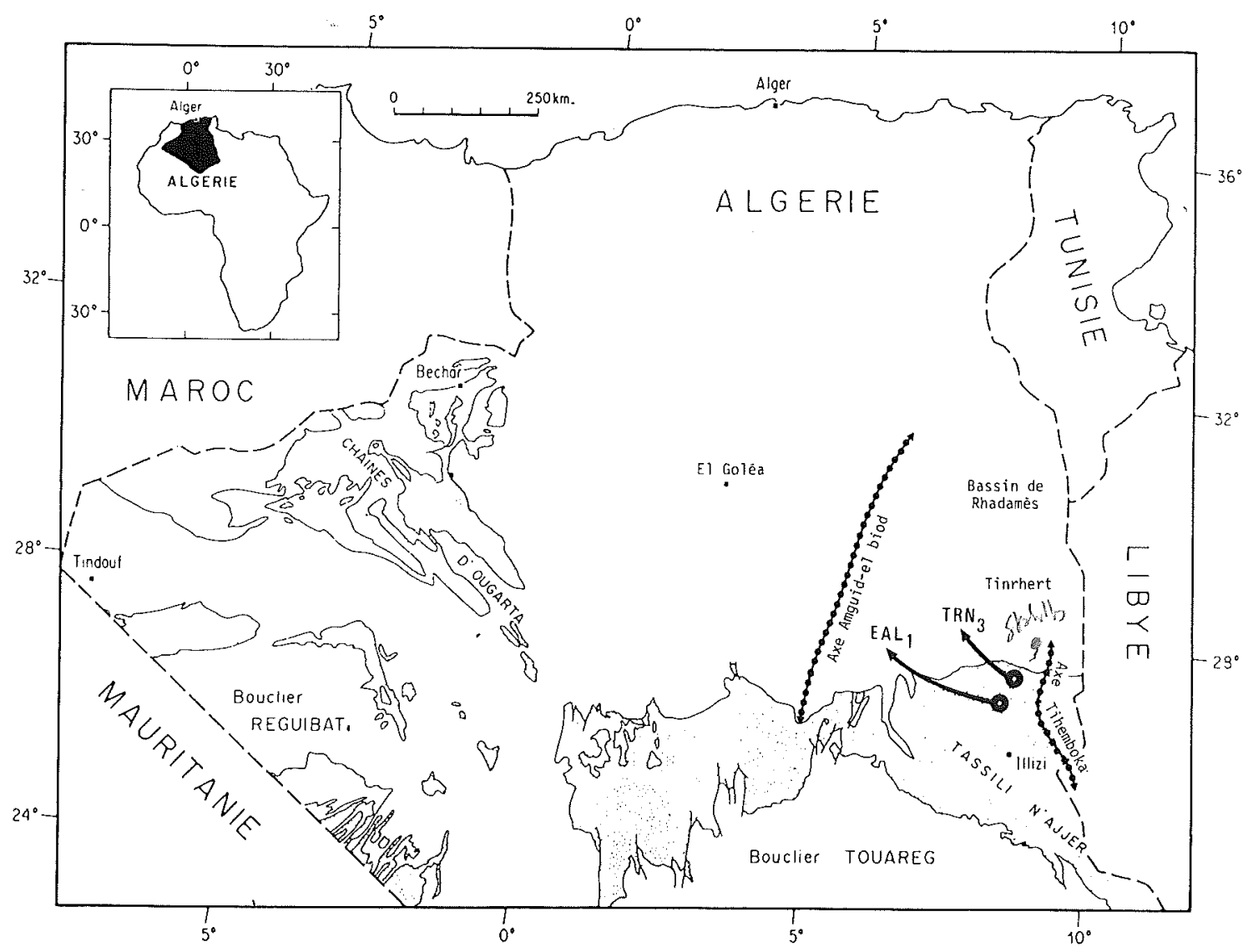

Fig. 1 - Localisation des puits TRN 3 et EAL 1 dans le bassin d'Illizi, SE de l'Algérie. Location of the wells TRN 3 and EAL 1 in the Illizi basin, SE Algeria. 


\section{SUCCESSION LITHOLOGIQUE DANS LE SILURIN SUPR ET LE DÉVONIEN}

\section{DU BASSIN D'ILLIZI}

Nous avons adopté la terminologie proposée par Jardiné \& Yapaudjian (1968) et Jardiné et alii (1974) pour les dépôts siluriens et dévoniens du bassin d'Illizi où, au-dessus des "Argiles à Graptolites", se succèdent respectivement les Formations de Méderba, de l'Oued Tifist, de Hassi Tabankort, d'Alrar et de Gazelle. Cette lithostratigraphie, élaborée à partir des données de plusieurs centaines de forages pétroliers du Tinrhert oriental et occidental, peut présenter quelques particularités régionales liées à la paléogéographie du bassin. On note ainsi l'importante réduction, voire l'absence, de certains termes de la succession, en particulier dans les Formations de l'Oued Tifist et de Hassi Tabankort (régression pouvant aboutir à une émersion temporaire s'accompagnant de phénomènes d'érosion). Dans les puits TRN 3 et EAL 1 , implantés dans la partie Sud-Est du bassin (fig. 1), la répétition de faciès assez semblables à divers niveaux de la séquence a posé quelques difficultés pour placer précisément les limites entre les divers termes habituellement distingués dans les Formations de l'Oued Tifist et de Hassi Tabankort.

1 - Formation DE MÉDERBa (jusqu’à $130 \mathrm{~m}$ de puissance).

Ce dépôt marin comprend un Membre inférieur gréso-micacé et un Membre supérieur argileux, à nombreuses intercalations gréso-quartziteuses. Seuls les 30 derniers mètres de la formation ont été echantillonnés dans le puits TRN 3 (intervalle compris entre 1665 et $1693 \mathrm{~m}$ ).

2 - FORMATION DE L'OUED TIFIST (jusqu'à $300 \mathrm{~m}$ de puissance).

Dans cet ensemble, les influences continentales s'affirment de plus en plus. Le Membre inférieur correspond à des grès verts montrant localement des caractères de dépôts estuariens, voire fluviatiles (Jardiné \& Yapaudjian 1968 ; Asses 1987). Le Membre moyen regroupe des grès chloriteux et des grès bioturbés alternant avec des shales verdâtres. Un horizon hématitique est connu dans ce membre. Le Membre supérieur englobe des grès grossiers à stratifications obliques, interprétés comme des dépôts fluviatiles (Jardi- né \& Yapaudjian 1968, fig. 8), et des shales gris-noir à niveaux gréseux lenticulaires.

Ces trois membres paraissent représentés dans le puits EAL 1 où seul le Membre supérieur a été échantillonné (carottes 54 à 56). Dans le puits TRN 3, en revanche, seul le Membre inférieur a été reconnu (carotte 16 ; intervalle compris entre 1650 et $1664,7 \mathrm{~m}$ ).

3 - FORMATION DE HASSI TABANKORT (jusqu'à 200 $\mathrm{m}$ de puissance).

Dans cette formation, correspondant apparemment aux unités 2 à 5 définies par Asses (1987), Jardiné \& Yapaudjian (1968) ont distingué :

- un Membre inférieur gréseux, à caractère fluviatile,

- un Membre moyen constitué d'alternances de siltstones et de grès fins et comportant un horizon à structure pseudo-bréchique,

- un Membre supérieur correspondant à des grès quartzitiques fins, à débris charbonneux.

Apparemment assez complète dans le puits EAL 1 (carottes 43 à 54 ; intervalle compris entre 1327 et $1428 \mathrm{~m})$, cette formation paraît réduite à son seul Membre supérieur dans le puits TRN 3 (carottes 12 à 15 ; intervalle compris entre 161.2 et $1650 \mathrm{~m}$ ).

4 - FORMATION D'ALRAR (jusqu'à $160 \mathrm{~m}$ de puissance).

Elle débute par des shales et des siltstones micacés gris noir (Membre inférieur), se poursuit par des alternances gréso-argileuses (Membre moyen) et s'achève par des grès massifs à nombreuses stratifications obliques (Membre supérieur).

Bien représentée dans les puits TRN 3 (de 1518 à $1612 \mathrm{~m}$; carottes 6 à 11 ) et EAL 1 (de 1220 à $1327 \mathrm{~m}$; carottes 30 à 42), cette formation montre des contacts assez francs par rapport aux formations encaissantes. 
5 - GROUPE DE GAZELle (= Formation de Gazelle, sensu Jardiné et alii 1974) (plus de $200 \mathrm{~m}$ de puissance) ;

La présence d'un horizon à Placodermes ("bonebed") coincidant avec un changement brutal de la composition du paléoplancton dans le puits TRN 1 (Boumendjel 1987) est interprétée ici comme la marque d'un important hiatus stratigraphique. Ceci nous conduit à séparer l'ensemble dénommé Formation de Gazelle par Jardiné et alii (1974) en deux unités dis- tinctes : la Formation de Gazelle inférieure et la Formation de Gazelle supérieure. Un horizon oolithique marque la base de la première qui correspond à une masse de shales gris ou noirs (intervalle compris entre 1328 et $1518 \mathrm{~m}$ dans le puits TRN 3), avec quelques intercalations calcareuses vers sa partie inférieure. $\mathrm{La}$ seconde débute avec l'horizon à Placodermes et présente ensuite des faciès gréseux (carottes 3 à 5 ; intervalle compris entre 1275 et $1328 \mathrm{~m}$ ); elle correspond à la régression fini dévonienne.

\section{RÉSULTATS BIOSTRATIGRAPHIQUES}

Pour éviter toute ambiguité nous préciserons ici les limites de Séries et d'Etages utilisées dans les discussions biostratigraphiques qui suivent. La Commission Internationale de Stratigraphie a déjà ratifié le choix des limites Ludlow-Pridoli, Pridoli-Lochkovien, Emsien-Eifelien et Givétien-Frasnien (cf. Basset 1985). Pour les autres, aucune décision définitive n'est encore adoptée ; nous utiliserons donc provisoirement les limites suivantes:

- Lochkovien-Praguien : apparition des véritables Nowakia acuaria, ce qui coïncide pratiquement avec les premiers Angochitina comosa (Chlupac et alii 1985).

- Praguien-Emsien : base de la Zone de Conodontes à dehiscens.

- Eifelien-Givétien : dans la Zone de Conodontes à ensensis, sachant que le calcaire de Givel, dans sa région type, débute vraisemblablement dans la partie supérieure de cette zone (cf. infra).

- Frasnien-Famennien : base de la Zone de Conodontes à triangularis inférieure.

\section{A - BIOSTRATIGRAPHIE DES MIOSPORES (S. Lobo-} ziak, Ph. Steemans \& M. Streel).

La distribution des échantillons fertiles dans le sondage TRN 3 est irrégulière et laisse en particulier, sans aucune donnée palynologique, des intervalles de l'ordre de 40 à $200 \mathrm{~m}$ (fig. 2).

Quelques-uns de ces échantillons ont livré une microflore relativement abondante et variée. Nous y avons recherché principalement les taxons, qu'à priori, nous croyons stratigraphiquement les plus utiles.
Un certain nombre d'espèces non utilisées sont cependant illustrées dans les planches photographiques (pl. 1-3).

1 - Assemblage de miospores du sommet de la Formation de Méderba (puits TRN 3, 1692 et 1693 m).

Nous y avons recueilli, en plusieurs exemplaires, Tholisporites chulus MC GREGOR var. chulus RICHARDSON \& LISTER, 1969 et Synorisporites verrucatus RICHARDSON \& LISTER, 1969.

Th. chulus var. chulus est connue depuis le Wenlockien jusqu'à l'Emsien, alors que $S$. verrucatus est notée du Downtonien inférieur à l'Emsien, mais est surtout présente dans le Downtonien.

Le peu de diversité spécifique dans ces échantillons suggère un niveau stratigraphique relativement ancien, à savoir Silurien, préalable à la période bien connue de grande diversification des formes dévoniennes. La même situation semble avoir été rencontrée par Massa \& Moreau-Benoit (1976) en Libye occidentale, dans leur zone palynologique $\mathrm{H}$, également datée Silurien ("Budnanien" supérieur).

2 - Assemblage de miospores du sommet de la Formation de Hassi-Tabankort (puits TRN 3, 1612,6 m).

Nous y avons reconnu :

Brochotriletes sp. cf. ? Perforosporites sp. 2 in Jardiné \& Yapaudjian (1968), Dictyotriletes emsiensis (ALLEN) Mc Gregor, 1973, Dictyotriletes cf. D. emsiensis 


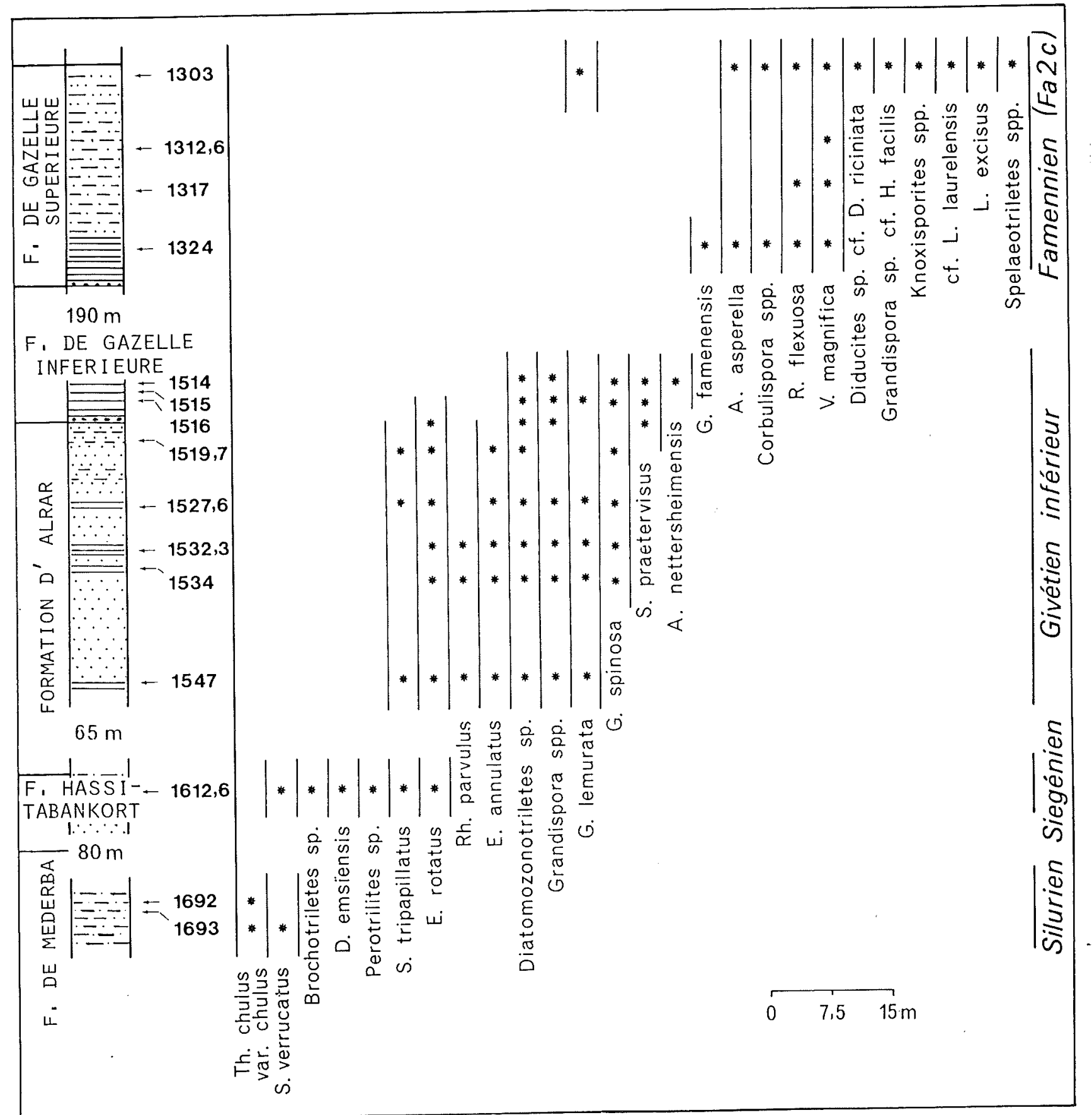

Fig. 2 - Distribution des principales miospores observées dans les échantillons fertiles du Silurien supérieur et du Dévonien du sondage TRN 3 (bassin d’lllizi, SE de l'Algérie).

Range of selected miospores in Late Silurian and Devonian fossiliferous samples from well TRN 3 (Illizi basin, SE Algeria). 
in Mc Gregor \& Camfield (1976), Emphanisporites rotatus (MC GREGOR) Mc Gregor, 1973, Perotrilites sp. cf. Zonotriletes 2 in Jardiné \& Yapaudjian (1968), Synorisporites tripapillatus RICHARDSON \& LISTER, 1969 et $S$. verrucatus.

Dans cet ensemble de taxons, $D$. emsiensis est connue comme représentant le niveau d'apparition le plus récent, à savoir le sommet du Lochkovien (zone BZ, Richardson et alii 1982; Streel et alii 1987). Son extinction se situe dans l'Emsien moyen.

L'absence de spores caractéristiques du Lochkovien supérieur, telle qu'entre autre, Emphanisporites zavallatus RICHARDSON et alii, 1982, ainsi que celle d'Emphanisporites annulatus MC GREGOR, 1961 dont le point d'apparition caractérise la base de l'Emsien, situe cet assemblage dans le Praguien.

Cet assemblage a également été vu par Jardiné \& Yapaudjian (1968) dans la Formation de Hassi Tabankort, au Tinrhert. Ces áuteurs l'attribuent à leurs zones palynologiques VI et VII qu'ils datent "Gedinnien-Siegenien". Plus récemment, Paris (1981) a plus ou moins attribué la zone VI à la zone à Chitinozoaires 31 qui, dans la région type de Bohême, est considérée d'âge Lochkovien supérieur (= Gedinnien supérieur).

Ces observations récentes sont donc compatibles avec l'âge que nous proposons.

3 - Assemblage de miospores du sommet de la Formation d'Alrar - base de la Formation de Gazelle in. férieure (puits TRN 3, 1514 à $1547 \mathrm{~m}$ ).

L'assemblage est ici nettement plus diversifié. Nous y avons retenu principalement : Diatomozonotriletes sp. cf. Acanthotriletes sp. 2 in Jardiné \& Yapaudjian (1968) et Diatomozonotriletes sp. in Massa \& MoreauBenoît (1976), Geminospora lemurata (BALME) Playford, 1983, Geminospora spinosa AlleN, 1965, Grandispora inculta ALLEN, 1965, G. libyensis MOREAUBENOIT, 1980, G. ? macrotuberculata (ARKHANGELSKAYA Mc Gregor, 1973, G. mammillata OWENS, 1971, G. rarispinosa MOREAU-BENOIT, 1980, G. velata (EISENACK) Mc Gregor, 1973, Rhabdosporites parvulus RICHARDSON, 1965 et Samarisporites praetervisus (NAUMOVA) Allen, 1965.

Dans cet ensemble de spores, les Geminospora du groupe lemurata (voir Playford 1983) et Rhabdosporites parvulus (voir Loboziak \& Streel 1980) sont considerées comme apparaissant le plus récemment, c'est-à-dire près de la limite Eifelien-Givétien. Jusqu'à présent, il n'y a pas de position reconnue internationalement pour la base du Givétien. La base du Calcaire de Givet, dans la région type de l'Ardenne, se situe probablement dans la partie supérieure de la Zone de Conodontes à Ensensis et serait proche de la base de la Zone à Varcus (Bultynck 1985). Ceci ne correspond pas à la base du Givétien en usage chez les auteurs allemands, qui est tracée entre les couches d'Ahbach et de Loogh dans la partie moyenne de la Zone à Ensensis (Ziegler 1979).

Les miospores ne sont hélas pas connues dans le Givétien type parce que les conditions sédimentaires n'y sont pas favorables. Geminospora lemurata apparaît pour la première fois, dans la région de l'Eifel, dans la Zone à Ensensis mais on ne sait pas avec certitude de quel côté de la limite Eifelien/Givétien proposée par les allemands (Streel et alii 1987). En revanche, il est probable que cette espèce $y$ est reconnue avant le niveau correspondant à la base du Calcaire de Givet. Par conséquent si ce dernier niveau etait retenu internationalement, l'apparition de G. lemurata se situerait dans la partie tout à fait superieure de l'Eifélien.

Etant donné que nous n'avons noté aucune Samarisporites triangulatus ALLEN, 1965, qui est une espèce bien connue à partir du Givétien "moyen", c'est-à-dire, dans le Boulonnais, au moins à partir de la Zone de Conodontes à Varcus moyenne, mais probablement avant cette zone (Streel et alii 1987), nous pouvons conclure pour cet ensemble à un âge transition Eifelien-Givétien. La distribution stratigraphique des Grandispora et de S. praetervisus est compatible avec ce résultat.

La présence du genre Diatomozonotriletes a déjà été précédemment notée au Givétien inférieur dans du matériel africain (Jardiné \& Yapaudjian 1968, Massa \& Moreau-Benoît 1970, Riegel in Paris et alii 1985). Il faut encore mentionner un assemblage voisin, d'âge "Givétien", dans les "Shales d'Accra" du Ghana (Bar \& Riegel 1974).

Plusieurs espèces habituellement rencontrées au sommet du Dévonien inférieur ou dans l'Eifélien ont été également identifiées : Ancyrospora nettersheimensis RIEGEL, 1973, E. annulatus, E. rotatus et $S$. tripapillatus. La persistance au Dévonien moyen et même supérieur des Emphanisporites était déjà connue. En revanche, c'est la première fois que l'on signale, près de la transition Eifelien-Givétien, des espèces telles que $S$. tripapillatus et $A$. nettersheimensis, cette dernière cependant est notée ici en un unique exemplaire. Elles 
pourraient traduire un remaniement consécutif à une émersion (cf. infra).

4 - Assemblage de miospores de la Formation de Gazelle supérieure) (puits TRN 3, 1303 à 1324 m).

Un nombre relativement élevé d'espèces y figure. Parmi elles, citons plus particulièrement : Auroraspora asperella (KeDO) Van der Zwan, 1979 ; Corbulispora sp. cf. Archaeozonotriletes amplectus (NAUMOVA) Kedo, 1963 ; Corbulispora subalveolaris (LUBER) Sullivan, 1964 ; Diducites sp. cf. Diaphanospora riciniata BALME \& HASSEL, 1962 ; Grandispora famenensis (NAUMOVA) Streel in Becker et alii 1974; Grandispora sp. cf. Hymenozonotriletes facilis KEDO, 1957 ; Knoxisporites dedaleus (NAUMOVA) Streel, 1977; K. hederatus (ISHENKO) Playford, 1963 ; cf. Leiozonotriletes laurelensis BALME \& HASSEL, 1962 ; Lophozonotriletes excisus NAUMOVA, 1953 ; Rugospora flexuosa (JUSHKO) Streel in Becker et alii 1974 ; Spelaeotriletes cf. crustatus HrGGS, 1975 ; S. granulatus (KEDO) Moreau-Benoît, 1979 ; S. cf. resolutus HigGS, 1975 et Vernciretusispora magnifica (MC GREGOR) Owens, 1971.

Toutes ces espèces apparaissent, au plus tôt, au Famennien et certaines même, telles que $G$. famenensis au Famennien supérieur ( $\mathrm{Fa} 2$ ) et $R$. flexuosa au $\mathrm{Fa}$ 2c.

L'absence de Retispora lepidophyta (KeDo) Playford, 1976, espèce qui marque par son abondance la partie sommitale du Fammenien ( $\mathrm{Fa} 2 \mathrm{~d}-\mathrm{Tn} 1 \mathrm{a})$, nous autorise à admettre pour cet assemblage un âge $\mathrm{Fa} 2 \mathrm{c}$.

Un assemblage comparable, daté aussi du même âge par la présence de $R$. flexuosa, a été identifié en Libye orientale par Streel in Paris et alii 1985. En Libye occidentale en revanche, la palynozone 10, mentionnée par Massa \& Moreau-Benoît (1976) comme appartenant à la base du Famennien moyen ne semble pas contenir cette espèce caractéristique. Le Fa $2 \mathrm{c}$ n'y serait donc pas représenté puisque la palynozone suivante (11) contient déjà $R$. lepidophyta.

En résumé, dans le puits TRN 3, nous observons 4 assemblages distincts de miospores datés Silurien, Praguien, transition Eifelien-Givétien et Fa 2c.

80 mètres sans aucun échantillon séparent l'assemblage Silurien de celui du Praguien et $65 \mathrm{~m}$, celui du Praguien de celui de la transition Eifelien-Givétien. L'analyse de 7 échantillons situés entre ces deux assemblages n'a livré que des spores banales et inutilisables. Enfin, 190 mettres séparent l'assemblage de la transition Eifelien-Givétien de celui du Fa 2c. Dans cet intervalle, l'examen de préparations provenant de "cuttings" à la recherche de formes caractéristiques, comme par exemple $S$. triangulatus, s'est avéré vain.

B - BIOSTRATIGRAPHIE DES CHITINOZOAIRES ET COMPARAISONS AVEC D'AUTRES FAUNES (K. BOUmendjel \& F. Paris), (pl. 4 et 5).

1 - Les assemblages de Chitinozoaires dans les Formations de Méderba et de l'Oued Tifist.

Les échantillons les plus anciens pris en compte dans cette étude proviennent du puits TRN 3 et ont été prélevés dans la partie supérieure de la Formation de Méderba, aux cotes 1692 et $1693 \mathrm{~m}$ (carotte 17). L'assemblage de Chitinozoaires observé dans ces niveaux est dominé par Gotlandochitina illiziensis BouMENDJEL, 1985 (jusqu'à $60 \%$ des taxons répertoriés ; fig. 3a). Cette espèce est accompagnée par Tanuchitina elenitae (CRAMER, 1964), T. sp. aff. cylindrica (TAugourdeau \& JeKHOWSKY, 1960), Cingulochitina serrata (TAUGOURDEAU \& JEKHOWSKY, 1960) et Ancyrochitina gr. ancyrea (EISENACK, 1931). C. serrata et T. elenitae ne coexistent que dans la partie moyenne du Ludlow (Cramer \& Diez 1978 ; Paris 1981), ce qui permet de dater la partie supérieure de la Formation de Méderba.

La limite entre cette unité et la Formation de l'Oued Tifist se placerait à $1665 \mathrm{~m}$ dans le puits TRN 3. Dans ce forage la partie terminale de la Formation de Méderba n'a pas été carottée et l'unique carotte prélevée dans la Formation de l'Oued Tifist est stérile. La datation de ces niveaux reposera donc uniquement sur l'exploitation biostratigraphique des Chitinozoaires recueillis dans les déblais de forages ("cuttings") entre 1650 et $1690 \mathrm{~m}$. Plectochitina carminae ? CRAMER, 1964 et divers Angochitina dont $A$. filosa EISENACK, 1955 ont été observés tout au long de cet intervalle. Il est exclu qu'il s'agisse de retombées de forage en provenance des niveaux sus-jacents puisque ceux-ci ne livrent pas d'exemplaires de Plectochitina. En toute rigueur, il est toutefois possible que ces formes soient issues des derniers niveaux $(1650 \mathrm{~m}$ à $1664 \mathrm{~m}$ ) de la Formation de l'Oued Tifist représentés dans ce puits ; auquel cas les exemplaires extraits des "cuttings" de la partie terminale de la Formation de Méderba (1665 m à $1690 \mathrm{~m}$ ) constitueraient des retombées. En dépit de cette incertitude, les biozones d'extension de $P$. carminae et des formes apparentees apportent des informations stratigraphiques. Ces 


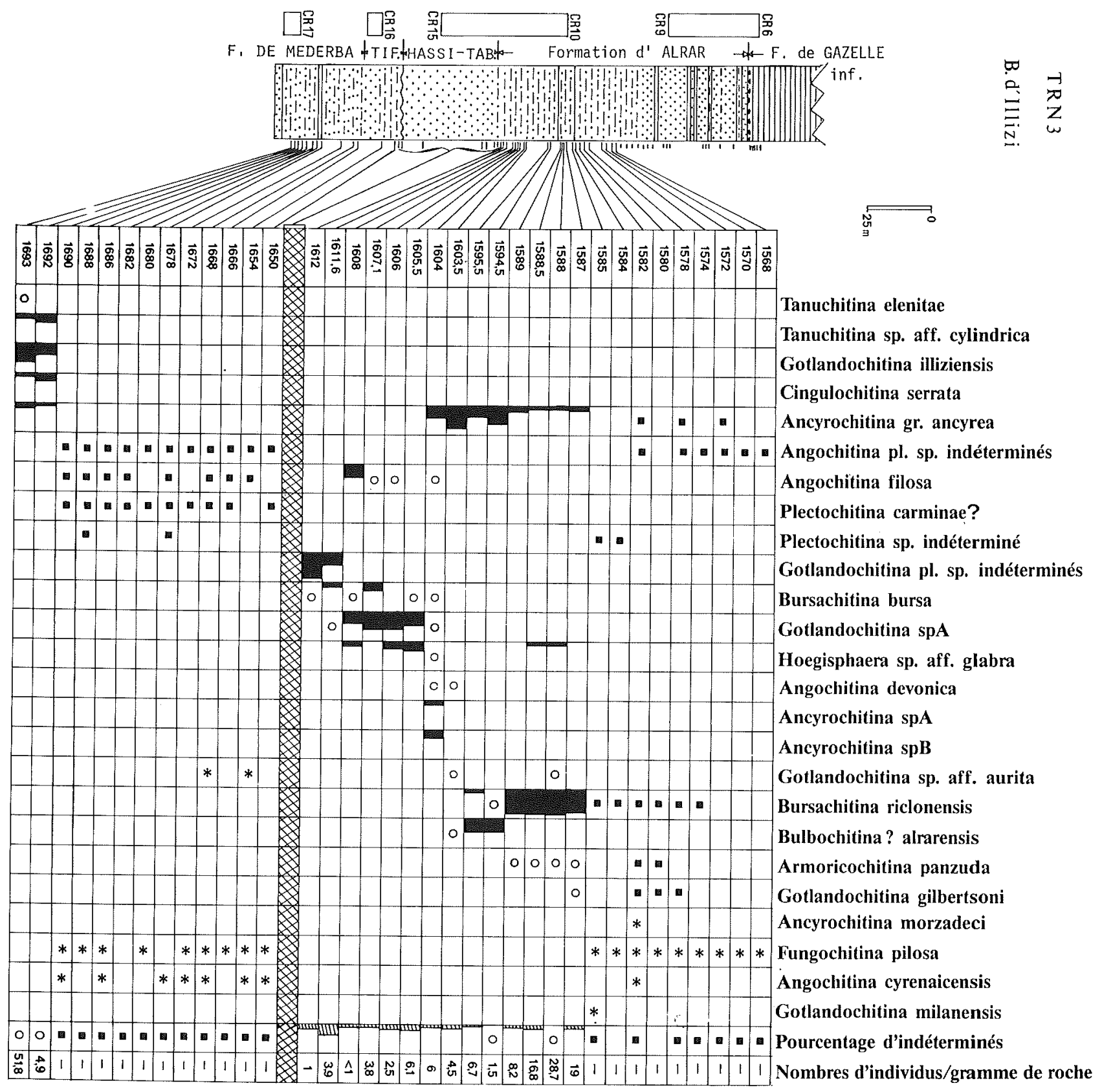

Fig. 3a - Distribution et fréquence relative des Chitinozoaires dans les Formations de Méderba et d'Alrar, sondage TRN 3 (bassin d'Illizi, SE de l'Algérie).

(Cercles : fréquences inférieures à $5 \%$; carrés noirs : spécimens provenant de đéblais de forage ; astérisques : "retombées" ; hachures et croisillons : échantillons stériles; voir fig. 5 pour le figuré de la colonne lithologique.

Range and relative abundance of the Chitinozoa from the Méderba and Alrar Formations, well TRN 3 (Illizi basin, SE Algeria).

(Circles: less than $5 \%$; black squares : individuals from cuttings; asterisks : cavate specimens; hatching : barren samples; see fig. 5 for the captions of the lithologic column). 


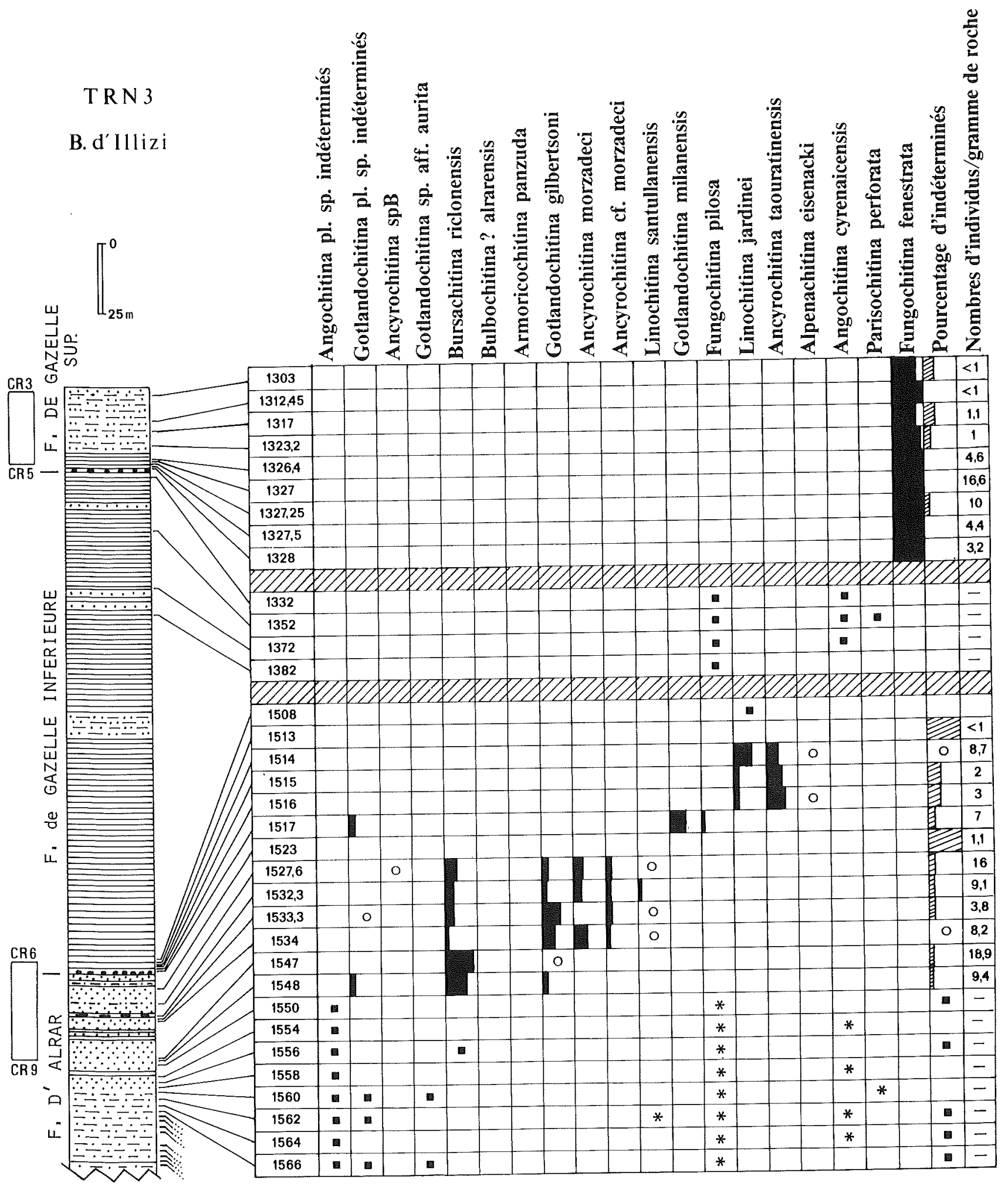

Fig. 3b - Distribution et fréquence relative des Chitinozoaires des Formations d'Alrar, de Gazelle inférieure et de Gazelle supérieure, sondage TRN 3 (bassin d'rllizi, SE de l'Algérie). (Même légenđe que la fig. 3a).

Range and relative abundance of the Chitinozoa from the Alrar, Lower Gazelle and Upper Gazelle Formations, well TRN 3 (Illizi basin, SE Algeria). (Same captions as fig. 3a) 
taxons, qui apparaissent dans le Ludlow, se poursuivent dans le Pridoli où elles sont généralement associées à l'un ou à l'ensemble des taxons suivants: Urnochitina urna (EISENACK, 1934), Pterochitina perivelata (EISENACK, 1937), Margachitina elegans (TAUGOUR. DEAU \& JEKHOWSKY, 1960) (cf. Cramer \& Diez 1978; Jaglin 1986 ; Boumendjel 1987 ; Paris 1981). Ces espèces n'ont pas été observées dans notre matériel. Ceci nous conduit à exclure un âge pridolien pour le Membre inférieur de la Formation de l'Oued Tifist.

Cette interprétation se trouve confirmée par un assemblage de Chitinozoaires recueilli au sommet du Membre supérieur de cette même formation, dans le puits EAL 1 (1428,5 m). Cet assemblage, qui renferme Ancyrochitina sp. aff. fragilis EISENACK, 1955 et Umochitina sp., est en effet dominé par Sphaerochitina patula $\mathrm{J}_{\mathrm{AGLIN}}$ 1986, espèce restreinte à la partie inférieure du Pridoli, en Libye et dans le Massif armoricain (Jaglin 1986).

En conséquence, dans le Sud-Est du bassin d'Illizi, le sommet de la Formation de Méderba et la majeure partie de la Formation de l'Oued Tifist appartiennent au Ludlow, tandis que le Membre supérieur de cette unité correspond à la partie inférieure du Pridoli.

\section{2 - Les termes de passage du Silurien au Dévonien.}

La Formation de Hassi-Tabankort, essentiellement constituée de grès blancs de haute énergie, a été largement carottée, tant dans le puits EAL 1 (carottes 43 à 54) que dans le puits TRN 3 (carottes 11 à 15), où elle paraît réduite à son seul Membre supérieur. Les nombreuses analyses palynologiques effectuées sur ce matériel à influence continentale marquée montrent une absence totale de Chitinozoaires tandis que les miospores apparaissent en abondance à la cote 1614,8 $\mathrm{m}$ dans le puits TRN 3, où elles coexistent avec des Acritarches dans les ultimes mètres de la formation (cotes 1614 et 1612,6 m) (Boumendjel 1987, fig. 73). Aucune datation directe de la Formation de HassiTabankort ne peut donc être fournie par les seuls Chitinozoaires. En revanche, ces microfossiles en permettant de dater le toit et la base des formations immédiatement en contact montrent que la Formation de Hassi-Tabankort s'est mise en place entre le Pridoli inférieur et la partie supérieure du Praguien. Les spores suggèrent même que la partie supérieure de la formation s'est déposée au Praguien (cf. supra).
3 - Assemblages de Chitinozoaires dans la Formation d'Alrar.

Un premier assemblage, correspondant à la biozone d'extension de Bursachitina bursa (TAUgourDEAU \& JEKHOwsKY, 1960), peut être défini à l'extrême base de la formation dans le puits TRN 3, où il s'étend sur 8 m, de 1604 m jusqu'à 1612 m (fig. 3a). Associées à l'espèce index on note diverses formes appartenant aux genres Ancyrochitina et Gotlandochitina, ainsi que Angochitina devonica EISENACK, 1955 et Hoegisphaera sp. aff. glabra STAPLIN, 1961. B. bursa décrite par Taugourdeau \& de Jekhowsky (1960) dans le Dévonien inférieur du Sahara est citée par Diez \& Cramer (1978) dans la formation des schistes de La Vid, rapportée par ces auteurs à l'Emsien s.l. En l'absence des Chitinozoaires caractéristiques des parties inférieure et moyenne du Praguien, et compte tenu de l'âge des niveaux sus-jacents, cet assemblage est rapporté ici à la partie supérieure du Praguien. Un âge Emsien basal ne peut toutefois être exclu. L'absence d'échantillons en position adéquate n'a pas permis de retrouver cet assemblage dans le puits EAL 1 (fig. 4).

Un second assemblage, caractérisé par l'extension de Bulbochitina ? alrarensis BOUMENDJEL, 1985, succède au précédent dans le puits TRN 3. L'espèce index y est associée à des exemplaires d'Ancyrochitina gr. ancyrea et aux premiers spécimens de Bursachitina riclonensis (PARIs, 1980) entre 1594,5 et 1603,5 m (fig. 3a). B. riclonensis débutant dans l'Emsien inférieur (Paris 1981), c'est donc un âge Emsien inférieur que nous retiendrons pour cet assemblage.

Un nouvel assemblage, coïncidant avec la biozone d'extension de Armoricochitina panzuda (CRAMER, 1964), peut être défini dans le puits TRN 3 entre les cotes 1580 et $1589 \mathrm{~m}$ incluses. L'espèce index y est associée à Bursachitina riclonensis (très abondante) et à $A$. gr. ancyrea. Quelques exemplaires de Gotlandochitina gilbertsoni (WOOD, 1974) ont d'autre part été observés vers le sommet de la biozone d'extension de $A$. panzuda (cf. fig. 3a). Le même assemblage a été reconnu dans le puits EAL 1 entre les cotes 1280,5 et $1310 \mathrm{~m}$ incluses, où l'on remarquera toutefois l'absence de représentants de $B$. riclonensis et l'abondance de Hoegisphaera sp. aff. glabra (cf. fig. 4). $A$. panzuda est une forme abondante dans la partie supérieure de la Formation des schistes de La Vid (Espagne) attribuée à l'Emsien supérieur (Diez \& Cramer 1978). A. panzuda d'autre part a été observée vers la base du Membre supérieur de la Formation de Teferguenite (Ougarta) où elle est associée à des Dacryoconarides de la limite Zlichovien-Dalejien (= 
- $339-$

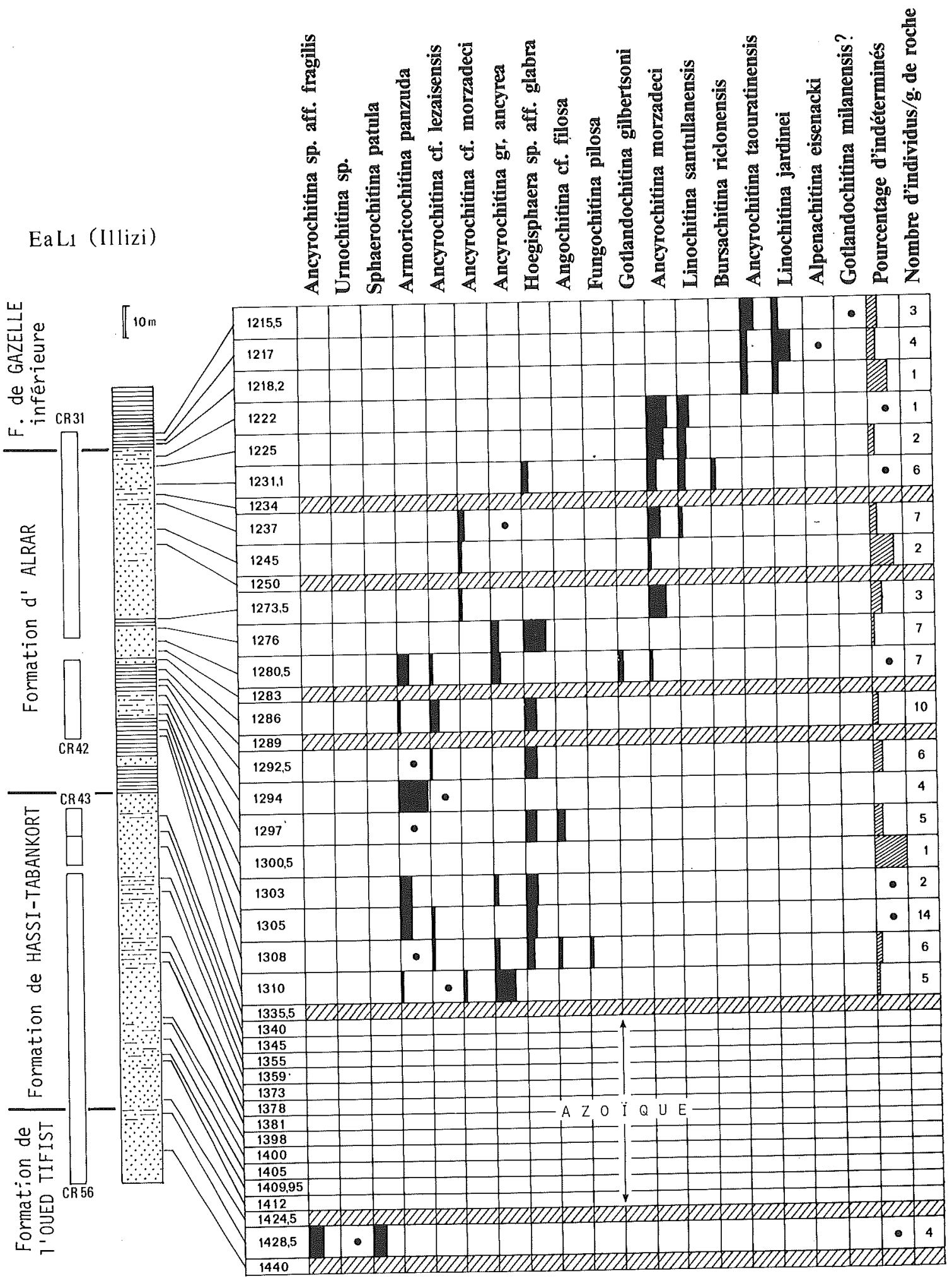

Fig. 4 - Distribution et fréquence relative des Chitinozoaires dans le Silurien supérieur et le Dévonien du sondage EAL 1 (bassın đ’mizi, SE de

l'Algérie).
(Cercles noirs : fréquence inférieure à $5 \%$; hachures : échantillons stériles; voir fig. 5 pour les figurés de la colonne lithologique).

Range and relative abundance of the Chitinozoa from the Late Silurian and the Devonian in well EAL 1 (Illizi Basin; SE Algeria). (Black circles : less than $5 \%$; hatching : barren samples; see fig. 5 for the captions of the lithologic column). 
base de l'Emsien supérieur) (Boumendjel 1987). Les niveaux livrant $A$. panzuda sont en conséquence attribués ici à la partie inférieure de l'Emsien supérieur.

Dans le puits TRN 3 la partie moyenne de la Formation d'Alrar n'a pas été carottée. Les déblais de forage disponibles entre 1550 et $1585 \mathrm{~m}$ n'apportent pas d'informations déterminantes au plan biostratigraphique. Ils permettent cependant de contrôler les extensions de $A$. panzuda et de l'assemblage reconnu dès la base de la carotte 9 (cotes 1527,6 à 1534 m) où Ancyrochitina morzadeci PARIS, 1980 et Linochitina santullanensis (DIEZ \& CRAMER, 1978) coexistent avec Gotlandochitina gilbertsoni (WOOD, 1974), Hoegisphaera sp. aff. glabra et avec les ultimes représentants atypiques de $R$. riclonensis (fig. 3). Une distribution similaire s'observe dans le puits EAL1 où l'association à A. morzadeci et L. Santullanensis s'étend entre 1232 et $1237 \mathrm{~m}$ (fig. 3b). On notera que dans le puits TRN 3, 8 $m$ de grès et siltstones à débris végétaux mais dépourvus de Chitinozoaires coiffent la formation.

A. morzadeci débute dans l'Emsien supérieur en Bretagne, dans la Formation des Marettes (Paris 1980). L. santullanensis a été décrite en Palencia (Espagne), dans un niveau rapporté par Cramer \& Diez (1978, p. 158 et 178) au Givétien inféricur en fonction de l'assemblage de miospores, Acritarches et Chitinozoaires qu'il renferme. L'extension stratigraphique et l'âge exact de $L$. santullanensis restent cependant à préciser. Les exemplaires typiques de $B$. riclonensis persistent au moins jusqu'au sommet de l'Emsien et sans doute dans la partie basale de l'Eifélien puisque l'espèce est encore abondante à la base de la Formation de Chefar el Ahmar, en Ougarta (Boumendjel 1987), sous les couches à Pinacites jugleri (Goddertz 1987). Gotlandochitina gilbertsoni paraît restreinte à la base de la Formation de Silica où elle a été décrite (Wood 1974) dans un niveau qui correspondrait à la partie inférieure de la Zone de Conodontes à Ensensis, si l'on admet les corrélations proposées par Driscoll et alii (1965). Dans l'état actuel des connaissances, les seuls Chitinozoaires ne permettent donc pas de fixer précisément l'âge du sommet de la Formation d'Alrar dans le Sud-Est du bassin d'Illizi. Quelques compléments d'information sont fournis par les macrofossiles. Jardiné et alii (1974) signalent en effet la présence de Paraspirifer cf. cultrijugatus dans le Membre moyen de la Formation d'Alrar, où ce Brachiopode précèderait les premiers exemplaires de $L$. santullanensis (= Urochitina sp. A in Jardiné \& Yapaudjian 1968). En Ougarta, Paraspirifer cultrijugatus disparaît au-dessus du "niveau coralligène" (Le Maitre 1952), marquant la base de la Formation de Chefar el
Ahmar, où il coexiste avec les derniers exemplaires de B. riclonensis (cf. Boumendjel 1987) vers le sommet de )'Emsien supérieur. Dans les affleurements de la bordure Sud du bassin d'Illizi, $P$. cultrijugatus est représenté (Chaumeau et alii 1961) à $1 \mathrm{~m}$ au-dessus d'un conglomérat marquant la limite entre les Formations de Hassi Tadjenout et de Aou Djilemt (sensu Legrand 1967). Un deuxième conglomérat, situé à la base de la Formation de Hassi Tadjenout, confirme le caractère instable de la sédimentation qui, dans les affleurements du Sud-Est du bassin d'Illizi, peut se traduire par une lacune des termes supérieurs du "Dévonien inférieur argilo-gréseux" et de la partie inférieure de la Formation de Aou Djilemt (cf. Legrand 1967, p. 265). Il est vraisemblables que de tels phénomènes, d'ampleur régionale, ont également été enregistrés dans les séquences recoupées par les puits TRN 3 et EAL 1. De fait, les "Grès massifs d'Alrar" (= Membre supérieur de la Formation d'Alrar), bien représentés dans le Nord du Tinrhert oriental, ne sont pas bien individualisés dans les puits TRN 3 et EAL 1. Ces "Grès massifs d'Alrar", parmi d'autres fossiles, livrent des Productellidés (Jardiné et alii 1974, p. 114), famille de Brachiopodes dont les premiers représentants apparaissent dans la base de l'Eifelien (Racheboeuf 1983). Ils surmontent d'autre part les niveaux à $L$. santullanensis (cf. Jardiné \& Yapaudjian 1968 ; tabl. 1). Compte tenu de la répartition de ce taxon dans les puits TRN 3 et EAL 1 (cf. fig. 3 et 4), il semble donc que le Membre supérieur de la Formation d'Alrar soit incomplet, ou non représenté, dans les sondages $d u$ Sud-Est du bassin d'Illizi. Cette lacune pourrait être annoncée par les grès et siltstones à débris de plantes qui, dans le puits TRN $3(1519,7$ à $1526 \mathrm{~m})$, terminent la formation, au-dessus de l'assemblage à $L$. santullanensis et $A$. morzadeci, et au-dessus des premiers exemplaires de $G$. lemurata de la limite Eifelien-Givétien (cf. supra). Le retour à une sédimentation marine franche ne s'effectue qu'avec l'horizon oolithique marquant la base de la Formation de Gazelle inférieure. Le hiatus stratigraphique reste toutefois de faible ampleur (intra Givétien inférieur) bien qu'il se marque par une nette rupture dans la composition des assemblages de Chitinozoaires.

La lacune la plus importante se place en fait sous l'assemblage à $L$. santullanensis et $A$. morzadeci. Elle correspondrait à une partie de l'Emsien superieur et à la quasi-totalité de l'Eifelien (voire l'extrême base du Givétien en fonction de la décision de la Commission Internationale de Stratigraphie concernant la position de la limite entre ces deux étages). Ce hiatus offre une explication à la présence de spores anté-givétiennes 
(ex. S. tripapillatus, A. nettersheimensis) au sein d'un assemblage à $G$. lemurata (cf. supra). En effet dans un contexte de sédimentation discontinue, des spores ont pu être resédimentées à la suite d'érosions locales de l'Emsien supérieur ou de l'Eifelien.

\section{4 - Assemblages de Chitinozoaires de la Formation de Gazelle inférieure.}

Dès la base de la formation, un assemblage à Linochitina jardinei BOUMENDJEL, 1985, Ancyrochitina taouratinensis BOUMENDJEL, 1985 et Alpenachitina eisenacki DUNN \& MILLER, 1964 succède au précédent dans les puits TRN 3 (carotte 6 , cotes 1514 à $1516 \mathrm{~m}$ ) et EAL 1 (carotte 31 ; cotes 1215,5 à 1218,2 m) (cf. fig. $3 \mathrm{~b}$ et 4$)$. A. taouratinensis et $L$. jardinei ne sont connues pour l'instant que dans le bassin d'Illizi (Boumendjel 1985) et n'apportent donc pas encore de précision d'ordre stratigraphique. En revanche $A$. eisenacki, bien que rare dans notre matériel, permet de discuter l'âge de la base de la Formation de Gazelle inférieure. Ce taxon, à large répartition géographique (cf. Jenkins \& Legault 1979), est très abondant dans l'Eifelien des USA, notamment dans la Formation de Jeffersonville (Wright 1980) appartenant à la Zone de Conodontes à Costatus costatus (Klapper \& Ziegler 1979) et dans la partie moyenne des Calcaires de Columbus de l'Ohio (Wright 1976). Ces derniers contiennent des Goniatites rappelant les formes de la Zone à Lateseptatus c'est-à-dire de la partie inférieure de l'Eifelien (House 1979). Le matériel type de $A$. eisenacki provient de la Formation d'Alpena, dans le Michigan, généralement mise en corrélation avec la partie supérieure du Membre Arkona de la Formation de Hamilton (Sandfort 1967). Cette espèce, qui est très rare dans le Membre Widder de la Formation de Hamilton (Legault 1973), pourrait donc persister jusque dans la partie moyenne de la Zone de Conodontes à Varcus, d'après les données de Klapper \& Ziegler (1979). La partie inférieure de la Formation de Gazelle inférieure ne paraît donc pas pouvoir être plus récente que le sommet de la Zone à Varcus moyenne.

Dans notre matériel, la rareté du genre Eisenackiti$n a$ doit être soulignée (2 spécimens, à $1514 \mathrm{~m}$, puits TRN 3 et à 1218,2 m, puits EAL 1, pl. 5, fig. 6). Parmi les ultimes représentants de ce genre, E. castor JANSONIUS, 1904 a été décrit dans la Formation de Hume dont la base et le sommet sont respectivement rapportés à la base des Zones de Conodontes à Australis et Ensensis (Klapper \& Ziegler 1979). Ce taxon est également cité en Libye (Massa \& Moreau-Benoît 1976;
Paris et alii 1985) dans des niveaux attribués à l'Eifelien supérieur pour les échantillons du Nord-Est de la Libye (Streel et alii 1988). En Amérique du Nord, $E$. castor ou des formes voisines, dont $E$. aranea (Urban, 1972), sont citées dans les Calcaires de North Vermon (Wright 1980), au sommet des Calcaires de Columbus (Wright 1976), dans la Formation de Silica (Wood 1974), dans les Membres Bell, Rockport quarry et Arkona de la Formation de Hamilton (Legault 1973) ainsi qu'à la partic inférieure du Membre Solon de la Formation de Cedar Valley (Urban 1972). Ces diverses formations se placent essentiellement dans un intervalle s'étendant de la Zone à Ensensis à la partie moyenne de la Zone à Varcus, d'après les données des Conodontes (Klapper \& Ziegler 1979 ; Ziegler et alii 1976). Dans le puits TRN 3, l'absence d'échantillons carottés au-dessus de notre assemblage à $A$. taouratinensis et $L$. jardinei, ne nous a pas permis de verifier la persistance des associations à Eisenackitina du Givétien inférieur dans la Formation de Gazelle inférieure, au-dessus de la cote $1514 \mathrm{~m}$. Cependant, Gotlandochitina milanensis (COLLINSON \& SCOTT, 1958) a été observée à l'extrême base de cette formation (cote $1517 \mathrm{~m}$ ). G. milanensis est connue dans diverses régions et notamment en Amérique du Nord ou elle devient abondante dans la partie moyenne du Membre Solon de la Formation de Cedar Valley (Collinson \& Scott 1958) au-dessus de la biozone d'acmé de Eisenackitina aranea (Urban 1972 ; fig. 9). Bien que ce type d'argumentation soit à utiliser avec précautions, il semblerait que la Formation de Gazelle inférieure débute au niveau des derniers représentants du genre Eisenackitina, c'est-à-dire dans le Givétien inférieur, au-dessous de la partie moyenne de la Zone de Conodonte à Varcus

Dans le bassin d'Illizi, l'ensemble à dominante argileuse constituant la Formation de Gazelle inférieure est rarement carotté et dans le puits TRN 3, seuls quelques échantillons de déblais de forage ont pu être analysés dans les cinquante derniers mètres de la formation (fig. 3). Trois espèces de Chitinozoaires, Fungochitina pilosa (COLLINSON \& SCOTT, 1958), Angochitina cyrenaicensis PARIS, 1988 et Parisochitina perforata BOUMENDJEL, 1985 sont identifiées dans ces déblais de forage, entre 1332 et $1382 \mathrm{~m}$. La possibilité de retombées est exclue, aucune de ces espèces n'étant présente dans les carottes de sondages analysées dans la Formation de Gazelle supérieure (cf. fig. 3b).

$P$. perforata n'est actuellement connue que dans le seul puits TRN 3 ; elle ne fournit donc aucun élément de datation. F. pilosa est largement répandue dans des formations du Givétien (cf. distribution in Jenkins \& 
Legault 1979). Ce taxon devient abondant vers le sommet du Membre Solon (Collinson \& Scott 1958) et dans le Membre Rapid de la Formation de Cedar Valley (Urban 1972). Dans la zonation des Conodontes, ces niveaux correspondent aux Zones à Varcus supérieure et à Hermanni-Cristatus inférieure (Ziegler et alii 1976). En Libye (bassin de Rhadamès), $F$. pilosa est citée dans la Formation Aouinet-Ouenine III (Massa \& Moreau-Benoît 1976), et prédomine dans des niveaux attribués au Givétien inférieur (Streel $e t$ alii 1988) dans le puits A 1-37 (N.E. de la Libye). $F$. pilosa n'a été observée ni dans le Frasnien de Belgique (Martin 1982) ou du Boulonnais (Paris 1988), ni dans les couches de passage du Givétien au Frasnien, en Montagne Noire (Paris \& Feist 1983). A. cyrenaicensis, décrite en Libye du N.E. (puits B 1-31) dans un niveau à Geminospora lemurata, rapporté au Givétien inférieur (Streel et alii 1988), n'a pas été observée dans le Frasnien de cette région. L'ensemble de ces éléments nous conduit à attribuer le sommet de la Formation de Gazelle inférieure au Givétien proparte (antérieur à la Zone de Conodontes à Disparilis).

\section{5 -. Assemblage de Chitinozoaires de la Formation de Gazelle supérieure.}

Dès la base de la Formation de Gazelle supérieure dans le puits TRN 3 , soit à 4 mètres au-dessus du dernier échantillon de déblais de forage livrant l'assemblage précédent, apparaît Fungochitina fenestrata (TAUGOURDEAU \& JEKHOWSKY, 1960). Cette espèce, relativement abondante dans les carottes 3 à 5 , (cotes 1303 à $1328 \mathrm{~m}$ ), constitue un assemblage monospécifique (fig. 3b).

F. fenestrata est une forme bien connue dans le Dévonien supérieur du Sahara algérien où elle apparaît comme l'ultime représentant des Chitinozoaires dans la région (Taugourdeau \& Jekhowsky 1960 ; Taugourdeau 1962). En Libye, dans le bassin de Rhadamès, $F$. fenestrata citée dès la Formation de Aouinet-Ouenine IV inférieure, disparaît au sommet de la Formation de Tahara, en même temps que les derniers représentants de Retispora lepidophyta (Massa \& Moreau-Benoît 1976). En terme de zonation de Conodontes, $F$. fenestrata s'éteint donc au Famennien terminal dans des niveaux correspondant à la Zone à Praesulcata (Streel et alii 1987).

En l'absence des Chitinozoaires du Frasnien supérieur et de la partie inférieure du Famennien, et conformément aux résultats fournis par les miospores, la Formation de Gazelle supérieure est donc attribuée au Famennien supérieur.

Il apparaît peu vraisemblable que le sommet du Givétien, le Frasnien et le Famennien inférieur puissent être condensés dans l'intervalle de quatre mètres (1328 à $1332 \mathrm{~m})$ séparant l'assemblage à $F$. fenestrata du précédent.

En effet, vers le sommet de la Formation de Gazelle inférieure, l'accroissement progressif de la proportion des miospores et microrestes végétaux au détriment des Acritarches et des Chitinozoaires (Boumendjel 1987) traduit une tendance régressive plutôt qu'un processus de sédimentation condensée qui se développe généralement en faciès plus pélagique ou distal. C'est donc une lacune que nous plaçons sous la Formation de Gazelle supérieure qui débute par un horizon légèrement ferrugineux, à fragments de Placodermes. Ce hiatus stratigraphique, précédant la transgression du Famennien supérieur, ne constitue pas un fait isolé dans le Sud-Est du Sahara. Des phénomènes similaires ont en effet été notés dans les affleurements orientaux de la bordure sud du bassin d'Illizi où la Formation du Djebel Illerène, d'âge Famennien supérieur, peut reposer jusque sur des terrains du Dévonien inférieur (Chaumeau et alii 1961 ; Legrand 1967). Cette lacune d'ampleur régionale a également été repérée dans un autre sondage du bassin d'Illizi (Abdeslam-Rhouighi 1986).

\section{CONCLUSIONS}

Les résultats de cette étude soulignent une nouvelle fois le caractère complémentaire des données des miospores et des Chitinozoaires dans la datation des séquences siluro-dévoniennes. Les miospores apportent en effet des données biostratigraphiques sur les dépôts très proximaux (ex. sommet de la Formation de Hassi Tabankort), où les Chitinozoaires sont absents. Ces derniers offrent eux-mêmes de précieux éléments de corrélations à longues distances, en domaine marin franc (Formations de Méderba, de Gazelle inférieure). 


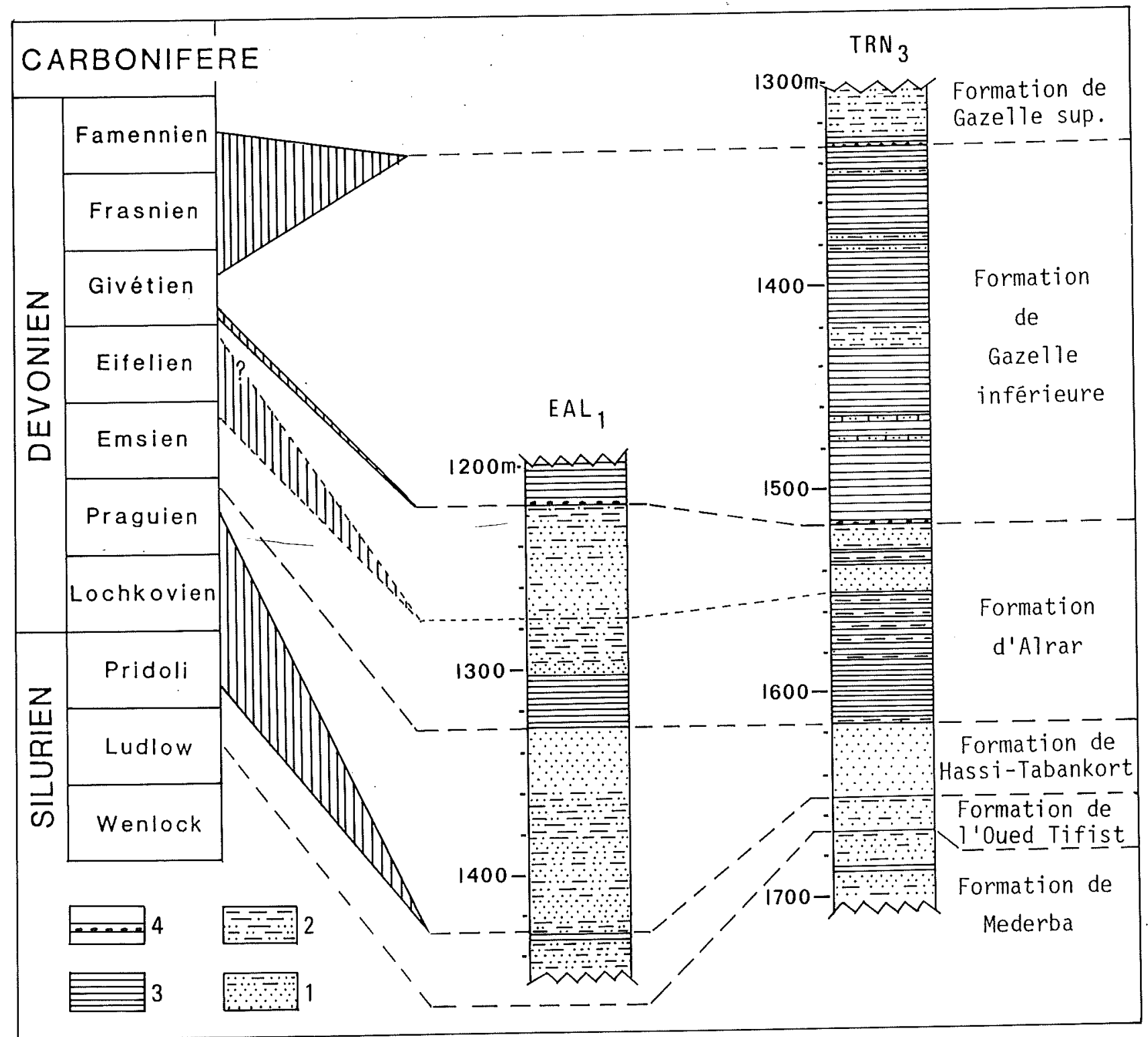

Fig. 5 - Attributions stratigraphiques et importance des lacunes observées dans les puits TRN 3 et EAL 1 (bassin d'lllizi, SE de l'Algérie). ( $1:$ grès $; 2:$ siltstones et grès ; $3:$ shales $; 4:$ niveaux ferrugineux oolithiques; hachures : hiatus sédimentaire).

Age assignment and importance of the stratigraphic gaps in wells TRN 3 and EAL 1 (Illizi basin ; S.E. Algeria). ( 1 : sandstones $; 2$ : siltstones and sandstones $; 3$; shales $; 4$ : oolithic ferruginous levels $;$ hatching : stratigraphic gap). 
Parallèlement à la datation des unités lithologiques représentées dans le Siluro-Dévonien du bassin d'Tllizi, l'utilisation conjointe des miospores et des Chitinozoaires nous a permis de mettre en évidence et (ou) d'évaluer l'ampleur de plusieurs hiatus sédimentaires (fig. 5). Certaines de ces lacunes (Pridoli à Praguien pro-parte) étaient connues de longue date. D'autres, en revanche, n'avaient pas encore été caractérisées (lacune d'une partie de l'Emsien et de l'Eifelien), où étaient-simplement suspectées (absence du Givétien supérieur, du Frasnien et du Famennien inférieur).

Les lacunes les plus importantes (Pridoli-Praguien pro-parte et Givétien-Famennien pro-parte), et les épisodes d'érosion qui peuvent leur être associés, sont de toute évidence liées à des mouvements de socle (rejeu de failles anciennes, épirogénie modérée ...) dont les effets sont d'autant plus perceptibles qu'ils affectent des aires épicontinentales à faible bathymétrie. De nombreux auteurs ont interprété le hiatus situé au passage Silurien-Dévonien comme une discordance calédonienne. Il s'agit en réalité de réajustements de socle propres à la marge nord du craton saharien, et sans liaison directe avec la fermeture du Iapetus, responsable de l'orogenèse calédonienne.

D'autres lacunes, localisées au sein même des formations (ex. : Formations de Hassi-Tabankort et d'Alrar) peuvent résulter du déplacement des chenaux distributeurs sur la plaine deltaïque. Ces migrations sont effet susceptibles d'entraîner une érosion locale des dépôts antérieurs. Cette interprétation s'accorde assez bien avec le développement de dépôts argilo-silteux, riches en débris végétaux, mais dépourvus de microfossiles marins, au-dessus de corps gréseux (ex. : partie supérieure de la Formation d'Alrar, dans le puits TRN 3).

Les corrélations régionales que nous avons proposées restent encore ponctuelles et sont limitées au seul matériel contemporain du Tinrhert et de Libye (cf. supra). En effet, pour le reste du Sahara, la distribution des Chitinozoaires du Praguien au Famennien n'a pas fait l'objet de publications détaillées (certaines données existeraient toutefois dans des rapports internes de sociétés pétrolières). Les possibilités de comparaisons régionales se révèlent également assez limitées au niveau des miospores. Ainsi, les assemblages décrits par Lanzoni \& Magloire (1969) dans le Sahara occidental, ou par Attar et alii (1980) dans le bassin d'Illizi, correspondent au Dévonien terminal et surtout au Carbonifère inférieur. Il s'agit donc d'un matériel plus jeune qui surmonte, éventuellement avec un léger recouvrement stratigraphique, la succession siluro-dévonienne que nous avons analysée dans le bassin d'Illizi. Celle-ci s'est en définitive révélée très incomplète et ne peut donc intervenir seule dans l'élaboration d'une biozonation des Chitinozoaires applicable à l'ensemble de la province nord-gondwanienne.

\section{Remerciements}

Les auteurs sont redevables à la SONATRACH (Algérie) qui a mis des échantillons de forage à leur disposition et a autorisé la publication des résul tats. Ils remercient également Mme M. Le Moigne
(Rennes) pour la frappe du texte, Mme D. Bernard (Rennes) pour la réalisation des figures et Messieurs $\mathrm{S}$. Jardiné ( $\mathrm{Pau}$ ) et $\mathrm{Ph}$. Taugourdeau (Paris) pour leurs remarques et suggestions.

\section{RÉFÉRENCES BIBLIOGRAPHIQUES}

ABDESLAM-RouighI F. (1986) - Premiers résultats biostratigraphiques (miospores, acritarches et chitinozoaires) concernant le Dévonien moyent supérieur du môle d'Ahara (Bassin d'Illizi, Algérie). Rev. Micropal., Paris, 29, 2 : 87-92.
ASSES A. (1987) - Analyse des diagraphies de forage, séquences sédimentaires et paléogéographie des séries argilo-gréseuses déposées au passage Silurien-Dévonien dans la synéclise est-saharienne. Thèse Univ. Pau, 331 p. (inédit). 
Attar A., Fournier J., Candillier A.M. \& CoQUEL R. (1980) - Etude palynologique du Dévonien terminal et du Carbonifère inférieur $\mathrm{du}$ bassin d'Illizi (Fort-Polignac) Algérie. Rev. Inst. Fr. Pétrole, Paris, 35 : 585-618.

BAR P. \& RIEGEL W. (1974) - Les microflores des séries paléozoïques du Ghana (Afrique occidentale) et leurs relations paléofloristiques. Sciences géologiques, Strasbourg, 27, 1-2 : 3958.

BASSET M.G. (1985) - Toward a "Common language" in stratigraphy. Episodes, Ottawa, 8, 2: 87-92.

BOUMENDJEL K. (1985) - Nouvelles espèces de Chitinozoaires dans le Silurien et le Dévonien du Bassin d'Illizi (S.E. du Sahara algérien). Rev. Micropal., Paris, 28, 3 : 155-166.

BOUMENDJEL K. (1987) - Les Chitinozoaires du Silurien supérieur et du Dévonien du Sahara algérien (cadre géologique, systématique, biostratigraphie). Thèse Univ. de Rennes I, 181 p. (inédit).

BULTYNCK P. (1985) - Introductory discussion for definition of the base of the Givetian. Internal Report, Subcommission on Devonian stratigraphy (IUGS) (unpublished).

Chaumeau J., Legrand P. \& Renaud A. (1961) Contribution à l'étude du Couvinien dans le bassin de Fort-Polignac (Sahara). Bull. Soc. géol. France, Paris, (7), 3: 449-456.

Chlupac I., LuKes P., PaRIS F. \& SCHONLAUB H.P. (1985) - The Lochkovian-Pragian boundary in the Lower Devonian of the Barrandian area, Czechoslovakia. Jb. Geol. B.A., Wien, 128 : 9 42.

Collinson C. \& SCOTT A.J. (1958) - ChitinozoanFaunule of the Devonian Cedar Valley Formation. Illinois State Geol. Surv., Urbana, 247 : 134.

CRAMER F.H. \& DIEZ M. (1978) - Iberian chitinozoans. l. Introduction and summary of Pre-Devonian data. Palinologia, Leon, num. ext. : 149201.

DIEZ M.d.C.R. \& CRAMER F.H. (1978) - Iberian chitinozoans. - II. Lower Devonian forms (La
Vid shales and equivalents). Palinologia, Leon, num. ext. : 203-217.

Driscoll E., Hall D.D. \& MusSMan D.G. (1965) Morphology and paleoecology of the brachiopod Leiorynchus kellogi HALL, Middle Devonian, Ohio, Michigan, Ontario. J. Paleontol, Tulsa, 38 : 916-935.

GODDERTZ B. (1987) - Devonische Goniatiten aus SW Algerien und ihre stratigraphische einordnung in die Conodonten-abfolge. Palaeontographica, Stuttgart, Abh. A, 197, 4-6 : 127-220.

House M.R. (1979) - Biostratigraphy of early Ammonoidea. In House M.R., SCRUTTON C.T. \& BASSET M.G. (eds). The Devonian System. Spec. Pap. Palaeont., London, 23 : 263-280.

JAGLIN J.C. (1986) - Nouvelles espèces de Chitinozoaires du Pridoli de Libye. Rev. Micropal., Paris, $29,1: 44-54$.

JARdiné S., Combaz A., Magloire L., Peniguel G. \& VACHEY G. (1974) - Distribution stratigraphique des Acritarches dans le Paléozoïque du Sahara Algérien. Rev. Palaeobot. Palynol., Amsterdam, 18 : 99-129.

JARDINÉ S. \& YAPAUDIIAN L. (1968) - Lithostratigraphie et Palynologie du Dévonien-Gothlandien gréseux du bassin de Polignac (Sahara). Rev. Inst. Fr. Pétrole, Paris, 13, 4 : 439-469.

JENKINS W.A.M. \& LEGAULT J.A. (1979) - Stratigraphic ranges of selected chitinozoa. Palynology, Dallas, $3: 235-264$.

KLAPPER G. \& ZIEGLER W. (1979) - Devonian Conodont biostratigraphy. In : HOUSE M.R., SCRUTTTON C.T. \& BASSET M.G. (eds). The Devonian System. Spec. Pap. Palaeont., London, 23 : 199-224.

LANZONI E. \& MAGLOIRE L. (1969) -Associations palynologiques et leurs applications stratigraphiques dans le Dévonien supérieur et le Carbonifère inférieur du Grand Erg occidental (Sahara algérien). Rev. Inst. Fr. Pétrole, Paris, 24 : 441-469.

LEGAUlT J. A. (1973) - Chitinozoa and Acritarcha of the Hamilton Formation (Middle Devonian), 
southwestern Ontario. Geol. Surv. Canada Bull., Ottawa, 221, 103 p.

LEGRAND Ph. (1967) - Le Dévonien du Sahara algérien. International Symposium on the Devonian System, Calgary : 245-284.

Le Maitre D. (1952) - La faune du Dévonien inférieur et moyen de la Saoura et des abords de l'Erg Djemel (Sud-Oranais). Mat. Cart. Géol. Algérie, Paléont., 12, $170 \mathrm{p}$.

LOBOZIAK S. \& STREEL M. (1980) -Miospores in Middle-Upper Frasnian to Famennian sediments partly dated by Conodonts (Boulonnais, France). Rev. Palaeobot. Palynol., Amsterdam, $34: 49-66$.

LOBOZIAK S. \& STREeL M. (1988) - Synthèse palynostratigraphique de l'intervalle Givétien-Famennien du Boulonnais. In "Le Dévonien deserques. Bas Boulonnais (N France)", D. BRICE (ed). Biostratigraphie du Paléozoïque, Brest, 7 : 71-77.

MARTIN F. (1982) - Acritarches et Chitinozoaires de la partie supérieure du Frasnien dans un affleurement au Nord immédiat de Frasnes (Belgique). Bull. Inst. r. Sci. nat. Belg., Sciences de la Terre, 54, 2 : 1-17.

Massa D. \& Moreau-Benoit A. (1976) - Essai de synthèse stratigraphique et palynologique du système Dévonien en Libye occidentale. Rev. Inst. fr. Pétrole, Paris, 31, 2 : 287-333.

Mc Gregor D.C. \& CAMFIELD M. (1976) - Upper Silurian ? to middle Devonian spores of the Moose River Basin, Ontario. Geol. Survey Bull., Ottawa, 263, 63 p.

PARIS F. (1980) - Les Chitinozoaires In : La tranchée de la Lézais, Emsien supérieur du Massif armoricain. Sédimentologie, paléontologie, stratigraphie. Mém. Soc. géol. minéral. Bretagne, Rennes, 24 : 55-75.

PARIS F. (1981) - Les Chitinozoaires dans le Paléozoïque du Sud-Ouest de l'Europe (Cadre géologique - Etude systématique - Biostratigraphie). Mém. Soc. géol. minéral. Bretagne, Rennes, 26, $492 \mathrm{p}$.

PARIS F. (1988) - Chitinozoaires du Frasnien du Boulonnais (Nord de la France). In : Le Dévo- nien de Ferques, Bas-Boulonnais, Nord de la France. D. BRICE (ed.). Biostratigraphie du Paléozoïque, Brest, 7 : 154-161.

PARIS F. \& FEIST R. (1983) - Preliminary investigations on organic walled microfossils (Chitinozoans) from the Givetian-Frasnian boundary in the Montagne Noire (Southern France). I.U.G.S., Subcommission on Devonian stratigraphy (Montagne Noire, September 1983), Reports, Rennes : 41-43.

PARIS F., RichaRdSON J.B., RIEGEL W., STREEL M. \& VANGUESTAINE M. (1985) - Devonian (Emsian-Famennian) Palynomorphs. In Palynostratigraphy of Northeast Libya (B.T. THUSU and B. OWENS eds.).J. micropaleontol, London, 4, 1 : 49-82.

PLAYFORD G. (1983) - The Devonian miospore genus Geminospora BALME, 1962 : a reappraisal based upon topotypic $G$. lemurata (type species). Mem. Ass. Australas Paleontols, Sydney, 1: 311-325.

RaCheboeuf P. (1983) - Productidina (Brachiopodes) dévoniens du Synclinorium médian armoricain. Bull. Soc. géol. minéral. Bretagne, Rennes, (C), 15, 2 : 149-167.

Richardson J.B., Streel M., Hassan A. \& SteeMANS P. (1982) - A new spore assemblages to correlate between the Breconian (British Isles) and the Gedinnian (Belgium). Ann. Soc. Géol. Belgique, 105 ; 136-143.

SANDFORT B.V. (1967) - Devonian of Ontario and Michigan. In OsWALD D.H. (ed.). International symposium on the Devonian system, Calgary, 1 : 973-999.

STReel M., Higgs K., Loboziax S., Riegel W. \& STEEMANS P. (1987) - Spore stratigraphy and correlation with faunas and floras in the type marine devonian of the Ardenne-Renish regions. Rev. Palaeobot. Palynol., Amsterdam, $50: 221-229$.

Streel M., Paris F., Riegel W. \& Vanguestaine M. (1988) - Acritarch, chitinozoan and spore stratigraphy from the middle and late Devonian subsurface of Northeast Libya. In B. THUSU \& B. OWENS (ed.) : (sous- presse). 
TAugourdeau Ph. (1962) - Associations de Chitinozoaires dans quelques sondages de la région d'Edjelé (Sahara). Rev. Micropal., Paris, 4, 4 : 229-236.

Taugourdeau Ph. \& Jeknowsky B. De (1960) Répartition et description des Chitinozoaires Siluro-Dévoniens de quelques sondages de la C.R.E.P.S., de la C.F.P.A. et de la S.N. Repal au Sahara. Rev. Inst. Fr. Pétrole, Paris, 15, 9 : 1199-1260.

URBAN J.B. (1972) - A reexamination of Chitinozoa from the Cedar Valley Formation of Iowa with observations on their morphology and distribution. Bull. Am. Paleont., Ithaca, $275: 1-44$.

Wood G.D. (1974) - Chitinozoa of the Silica Formation (Middle Devonian, Ohio) : vesicle ornamentation and paleoecology. Mich. st. Univ. Museum Publ. paleont. Ser., East Lansing, 1 : 131-144.
WRIGHT R.P. (1976) - Occurrence, stratigraphic distribution and abundance of Chitinozoa from the Middle Devonian Columbus Limestone of Ohio. Ohio J. Sc., Marion, 76, 5 : 214-224.

WrIGHT R.P. (1980) - Middle Devonian Chitinozoa of Indiana. Sp. Rep. geol. Surv. Indiana, Dept. Nat. Res., Bloomington, $18: 1-24$.

ZIEGLER W. (1979) - Historical subdivisions of the Devonian. In HOUSE M.R., SCRUTTON C.T. \& BASSET M.G. (eds.). The devonian System. Spec. Pap. Palaeont., London, 23 : 23-47.

ZIEGLER W., KLAPPER G. \& JOHNSON J.G. (1976) Redefinition and subdivision of the varcusZone (Conodonts, Middle - ? Upper Devonian) in Europe and North America. Geologica Palaeont., Marburg, 10 : 109-140.

Manuscrit déposé le 27.01.1988

Manuscrit définitif reçu le 14.03.1988 


\section{PLANCHE 1}

\section{Miospores du sondage TRN 3}

(Grossissement : x 500)

Fig. 1 - Perotrilites sp. cf. Zonotriletes 2 in Jardiné \& Yapaudjian, 1968. Lame (2), 1612,6 m (J 48/3).

Fig. 2 - Brochotriletes sp. cf. ? Perforosporites sp. 2 in Jardiné \& Yapaudjian, 1968. Lame (1), 1612,6 m (J 46/4).

Fig. 3 - Dictyotriletes emsiensis (Allen) Mc Gregor, 1973. Lame (1), 1612,6 m (F 41).

Fig. 4, 5 - Tholisporites chulus (Cramer) Mc Gregor var. chulus Richardson \& Lister, 1969.

4 - Lame (1), 1693 m (P 41/4). 5 - Lame (1), 1692 m (P 50/2).

Fig. 6 - Synorisporites verrucatus Richardson \& Lister, 1969. Lame (1), 1612,6 m (0 33).

Fig. 7, 8 - Synorisporites tripapillatus Richardson \& Lister, 1969.

7. Lame (1), 1612,6 m (P 46).8 - Lame (1), 1612,6 m (H 47/1).

Fig. 9, 10 - Diatomozonotriletes sp. cf. Acanthotriletes sp. 2 in Jardiné \& Yapaudjian, 1968.

9 - Lame (1), $1514 \mathrm{~m}$ (N 42/1). 10 - Lame (1), $1514 \mathrm{~m}$ (Q 27).

Fig. 11, 12. - Emphanisporites annulatus Mc Gregor, 1961.

11 - Lame (1), 1527,6 m (D 48). 12 - Lame (1), 1534 m (F 33/4).

Fig. 13, 14 - Emphanisporites rotatus (Mc Gregor) Mc Gregor, 1973.

13 - Lame (1), 1532,3 m (Q 40/3). 14 - Lame (1), 1532,3 m (H 39).

Fig. 15 - Retusotriletes rotundus Lele \& Streel, 1969.

Lame (1), 1303 m (E 34/2).

Fig. 16. - Rhabdosporites parvulus Richardson, 1965.

Lame (NL), 1532,3 m (Q 48).

Fig. 17 - Grandispora inculta Allen, 1965.

Lame (1), 1532,3 m (R 46).

Fig. 18, 19 - Geminospora lemurata (Balme) Playford, 1983.

18 - Lame (1), 1527,6 m (U 32/2). 19 - Lame (1), 1532,3 m (F 49/3).

Fig. 20. - Geminospora spinosa Allen, 1965.

Lame (1), $1534 \mathrm{~m}$ (T 47).

Fig. 21 - Dibolisporites cf. gibberosus (Naumova) Richardson, 1965.

Lame (NL), 1519,7 m (V 36).

Fig. 22. - Samarisporites sp.

Lame (2), $1514 \mathrm{~m}$ (D 43).

Fig. 23 - Bullatisporites bullatus Allen, 1965.

Lame (1), $1534 \mathrm{~m}$ (C 40).

Fig. 24 - Dibolisporites echinaceus (Eisenack) Richardson, 1965.

Lame (1), 1516 m (H 35). 
Geobios

no 21 , fasc. 3

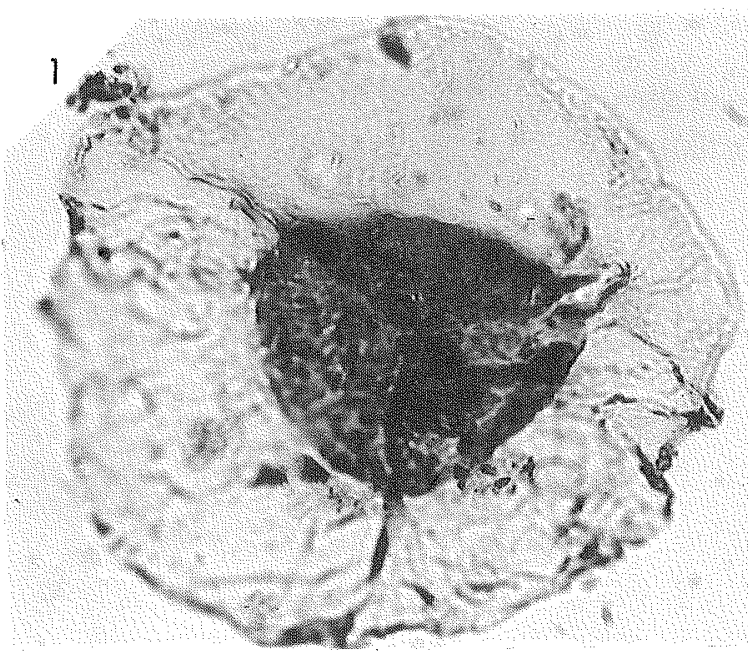

K. Boumendjel, S. Loboziak, F. Paris, P. Steemans \& M. Streel
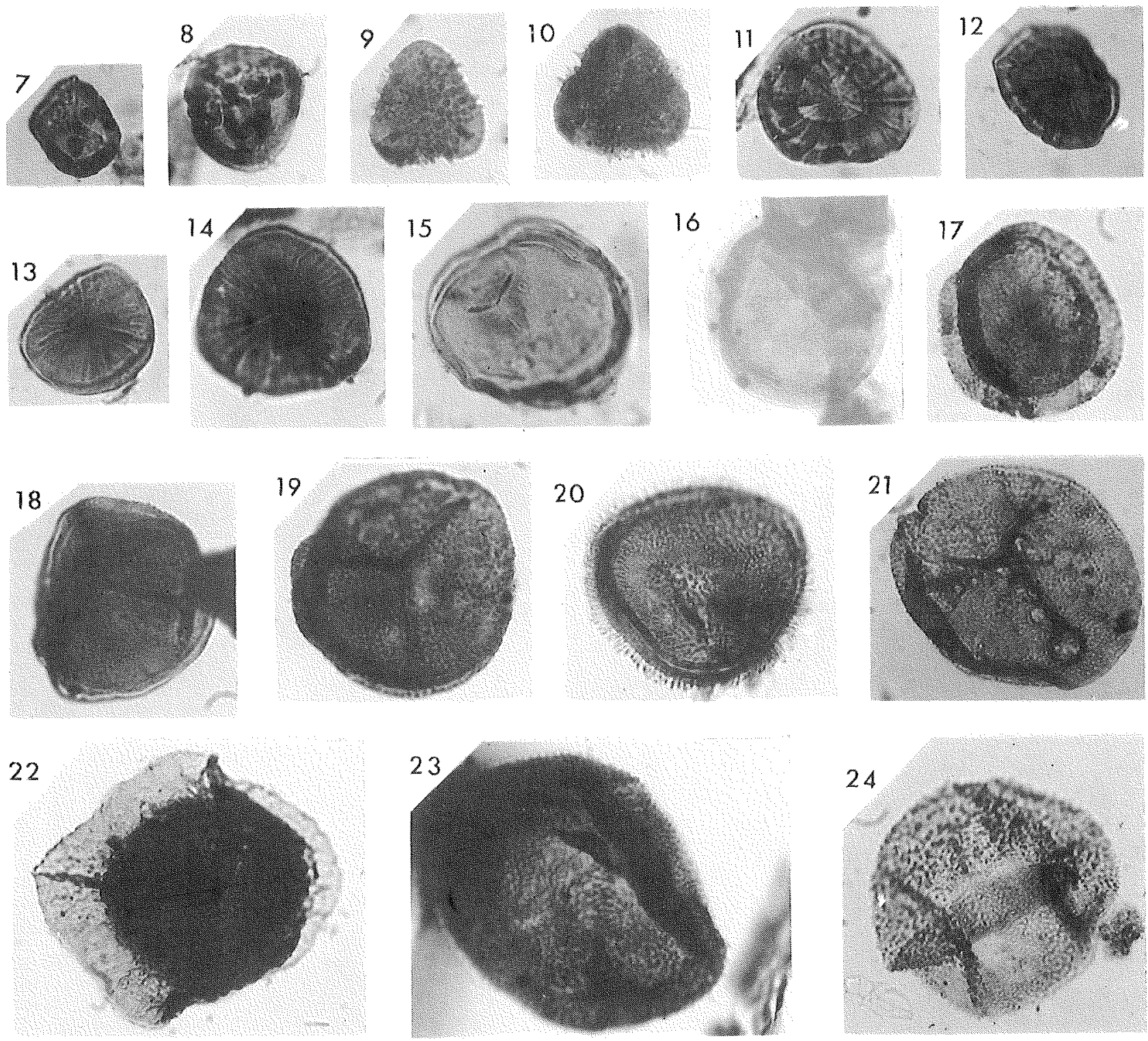


\section{PLANCHE 2}

\section{Miospores du sondage TNR 3}

(Grossissement : $\times 500$ )

Fig. 1 - Grandispora libyensis Moreau-Benoît, 1980.

Lame (2), 1514 m (Y 43/1).

Fig. 2. - Grandispora rarispinosa Moreau-Benoît, 1980.

Lame (3), 1516 m (P 42).

Fig. 3 - Grandispora ? macrotuberculata (Arkhangelskaya) Mc Gregor, 1973.

Lame (2), 1527,6 m (W 40).

Fig. 4 - Ancyrospora langii (Taugourdeau-Lantz) Allen, 1965.

Lame (NL), 1303 m (M 55/2).

Fig. 5 - Ancyrospora nettersheimensis Riegel, 1973.

Lame (2), $1514 \mathrm{~m}$ (G 36/4).

Fig. 6 - Grandispora famenensis (Naumova) Streel in Becker et alii, 1974.

Lame (1), $1324 \mathrm{~m}(\mathrm{~J} / 47)$.

Fig. 7 - Retusotriletes incohatus Sullivan, 1964.

Lame (1), 1303 m (H 40).

Fig. 8 - Corbulispora subalveolaris (Luber) Sullivan, 1964.

Lame (NL), $1303 \mathrm{~m}$ (H 42).

Fig. 9 - Corbulispora sp. cf. Archaeozonotriletes amplectus (Naumova) Kedo, 1965.

Lame (NL), $1303 \mathrm{~m}$ (X 34/2). 

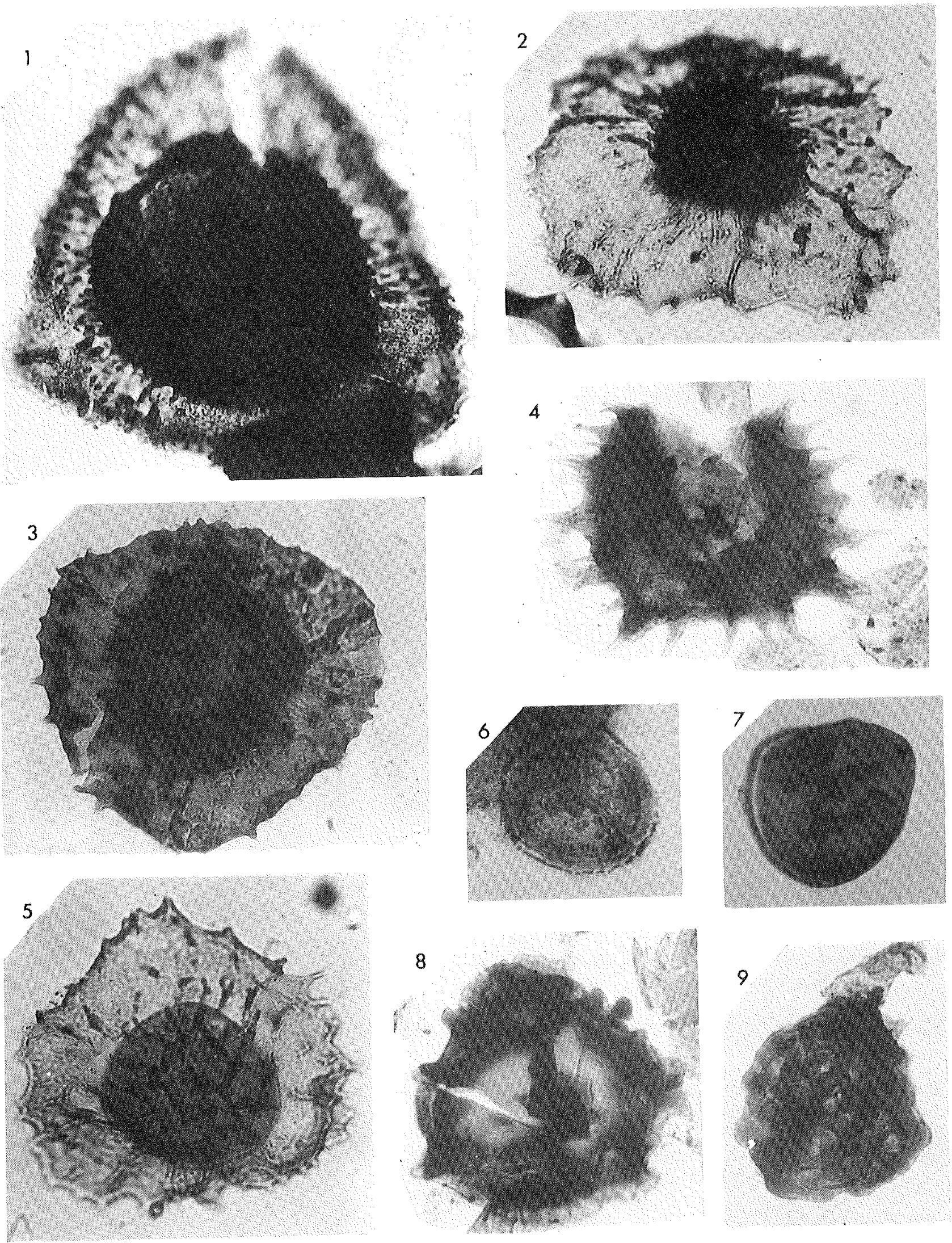


\section{PLANCHE 3}

\section{Miospores du sondage TRN 3}

(Grossissement : $x$ 500)

Fig. 1, 2 - Samarisporites praetervisus (Naumova) Allen, 1965.

1. Lame (2), $1514 \mathrm{~m}$ (H 45/2). 2. Lame (3), $1516 \mathrm{~m}$ (U 47).

Fig. 3 - Grandispora velata (Eisenack) Mc Gregor, 1975.

Lame (NL), 1519,7 m (U 37/1).

Fig. 4. - Grandispora mammillata Owens, 1971. Lame (1), 1519,7 m (L 35).

Fig. 5, 6 - Spelaeotriletes cf. crustatus Higgs, 1975.

5 - Lame (NL), 1303 m (Y 38). 6 - Lame (NL), 1303 m (L 50).

Fig. 7 - Knoxisporites dedaleus (Naumova) Streel, 1977.

Lame (1), 1303 m (K 34/2).

Fig. 8 - Knoxisporites hederatus (Ishenko) Playford, 1963, Lame (1), 1303 m (L 31).

Fig. 9 - Knoxisporites sp.

Lame (NL), 1303 m (S 38/3).

Fig. 10 - Verruciretusispora magnifica (Mc Gregor) Owens, 1971. Lame (NL), 1303 m (E 49/3).

Fig. 11 - Spelaeotriletes cf, resolutus Higgs, 1975. Lame (NL), $1303 \mathrm{~m}$ (R 38).

Fig. 12 - Aurorospora asperella (Kedo) van der Zwann, 1979.

Lame (1), $1303 \mathrm{~m}$ (Q 42).

Fig. 13 - Spelaeotriletes granulatus (Kedo) Moreau-Benoît, 1979. Lame (NL), $1303 \mathrm{~m}$ (X 30).

Fig. 14 - Lophozonotriletes excisus Naumova, 1953.

Lame (1), $1303 \mathrm{~m}$ (W 36).

Fig. 15 - Archaeozonotriletes variabilis (Naumova) Allen, 1965.

Lame (1), $1303 \mathrm{~m}$ (J 34/2).

Fig. 16 - Diducites sp. cf. Diaphanospora riciniata Balme \& Hassel, 1962.

Lame (NL), $1303 \mathrm{~m}$ (T 46/1).

Fig. 17 - cf. Leiozonotriletes laurelensis Balme \& Hassel, 1962. Lame (NL), $1303 \mathrm{~m}$ (S 40).

Fig. 18 - Rugospora flexuosa (Jushko) Streel in Becker et alii, 1974. Lame (NL), $1303 \mathrm{~m}$ (O 47/1).

Fig. 19 - Grandispora sp. cf. Hymenozonotriletes facilis Kedo, 1975. Lame (NL), 1303 m (R 37/2). 
Geobios

no 21 , fasc. 3
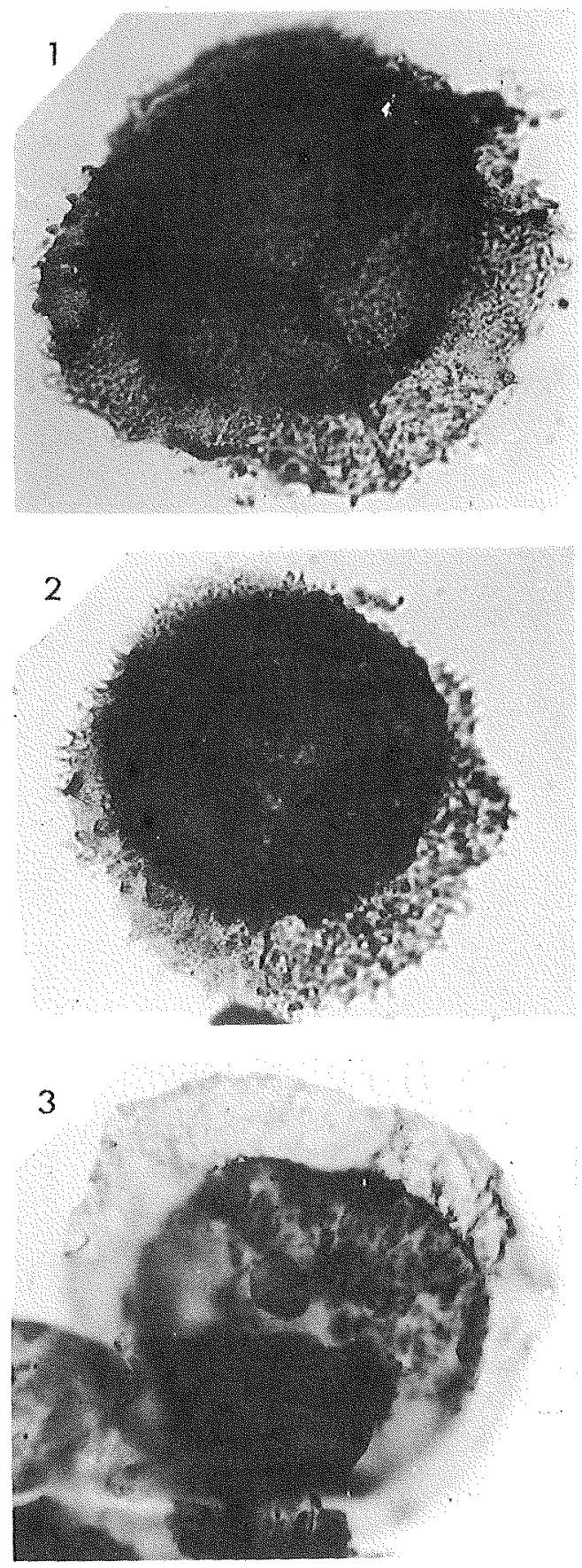

10
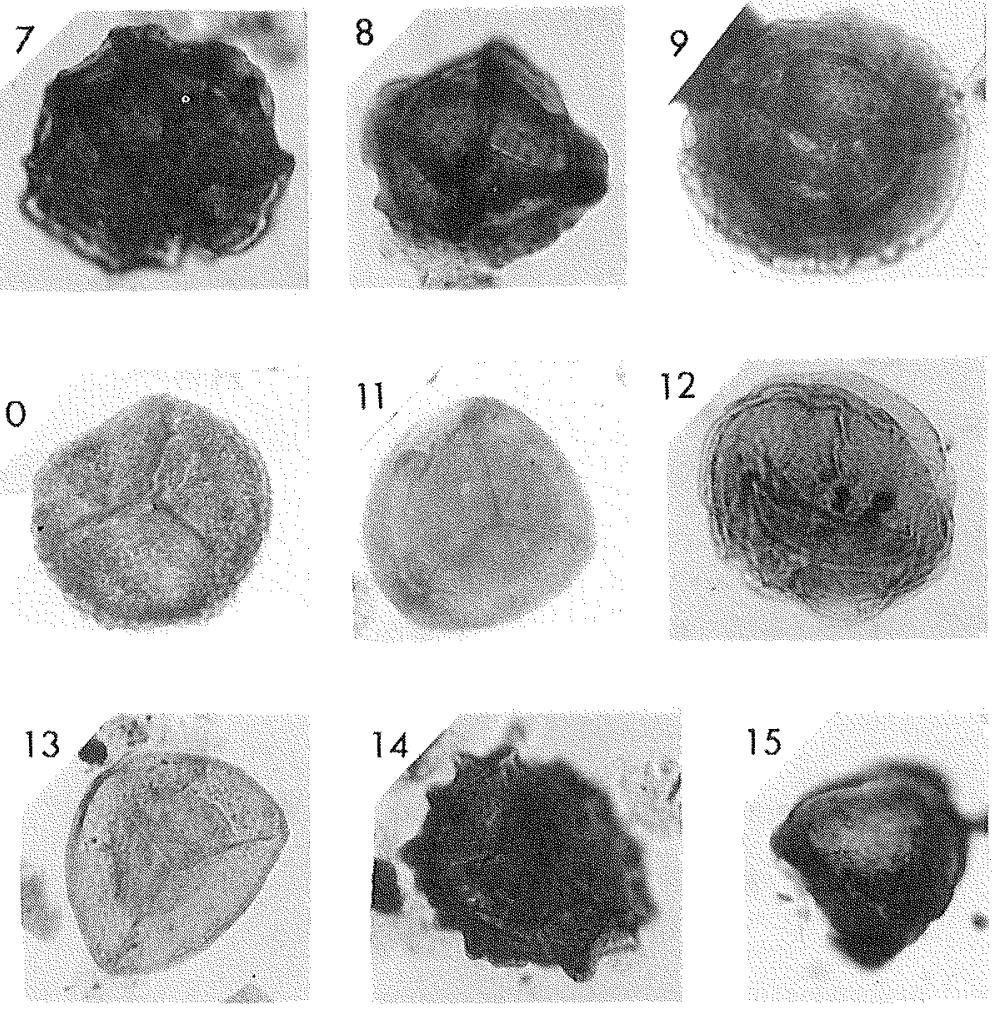
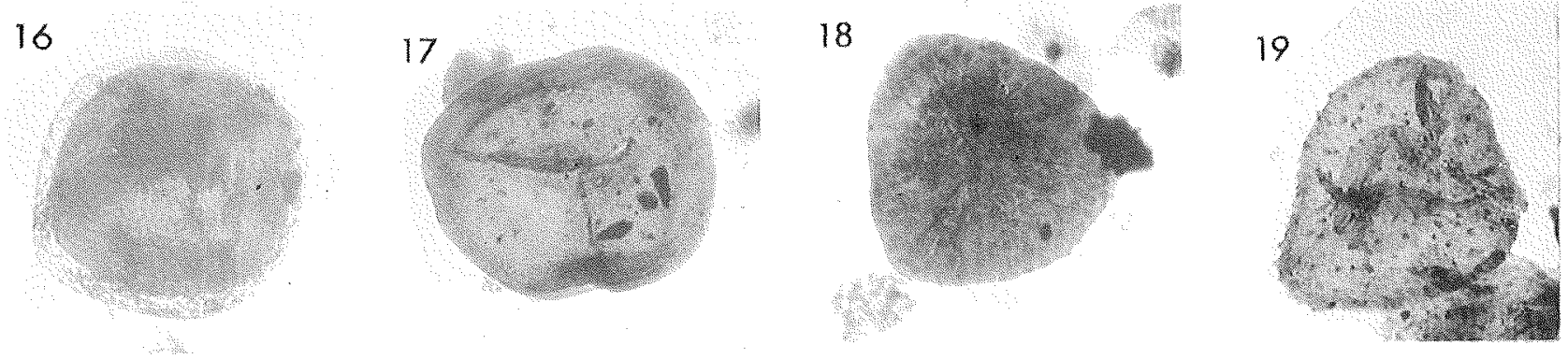


\section{PLANCHE 4}

\section{Chitinozoaires des sondages TRN 3 et EAL 1}

Fig. 1 - Gotlandochitina illiziensis Boumendjel, 1985, (x 400).

Sondage TRN 3, 1693 m, IGR 60230 (Q. 39/4).

Fig. 2a-b - Sphaerochitina patula Jaglin, 1986

2a - (x 400), sondage EAL 1, 1428,5 m, IGR 60342 (M. 34/1).

$2 \mathrm{~b}$ - ( $\mathrm{x} 1500)$, détail de l'ornementation du même spécimen.

Fig. 3 - Tanuchitina elenitae (Cramer, 1964).

(x 300), sondage TRN 3, 1693 m, IGR 60230 (P.36).

Fig. 4 et 5 - Bulbochitina alrarensis Boumendjel, 1985.

Sondage TRN 3, 1595 m, IGR 60254.

4 - (x 300), (K. 31), spécimen en vue orale.

5 - (x 300), (S. 35/4), spécimen en vue aborale.

Fig. 6 - Bursachitina bursa (Taugourdeau \& Jekhowsky, 1960).

(x 500), sondage TRN 3, 1607,1 m, IGR 60238 (H. 41).

Fig. 7 - Armoricochitina panzuda (Cramer, 1964).

(x 450), sondage EAL 1, 1294 m, IGR 60337 (N. 36/2).

Fig. 8 - Ancyrochitina morzadeci Paris, 1980.

(x 300), sondage TRN 3, 1534 m, IGR 60299, (P. 42).

Fig. 9 et 10 - Linochitina santullanensis (Diez \& Cramer, 1978).

Sondage TRN 3, 1534 m, IGR 60299.

$9-(\times 350),(0.39)$.

$10-(\mathrm{x} 300),(0.40 / 4)$. 
Geobios

$\mathrm{n}^{\mathbf{9}} \mathbf{2 1}$, fasc. 3

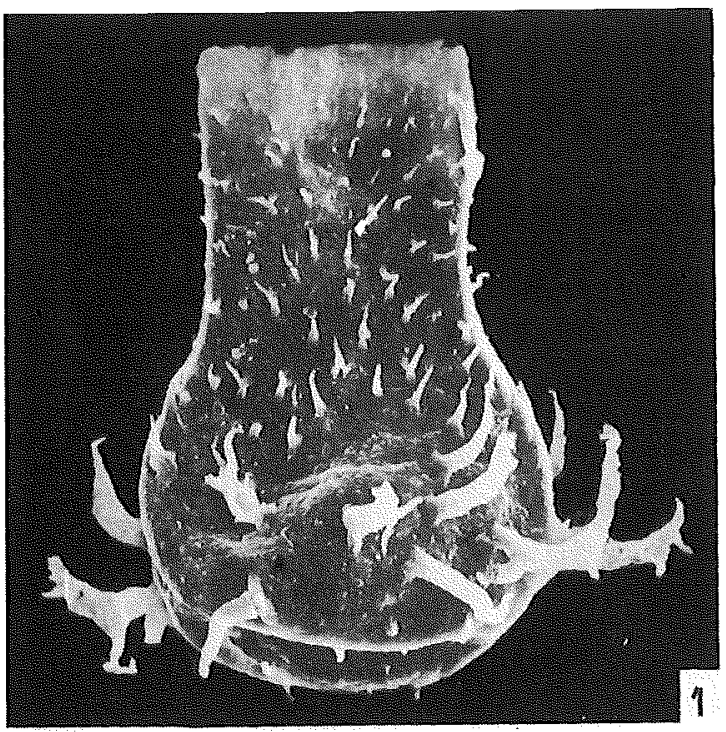

2. 1 (

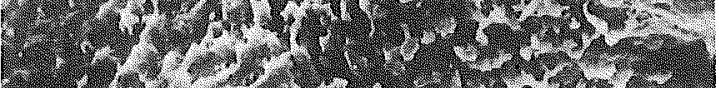

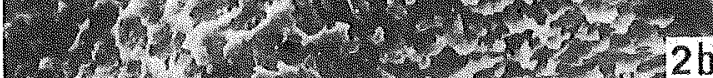
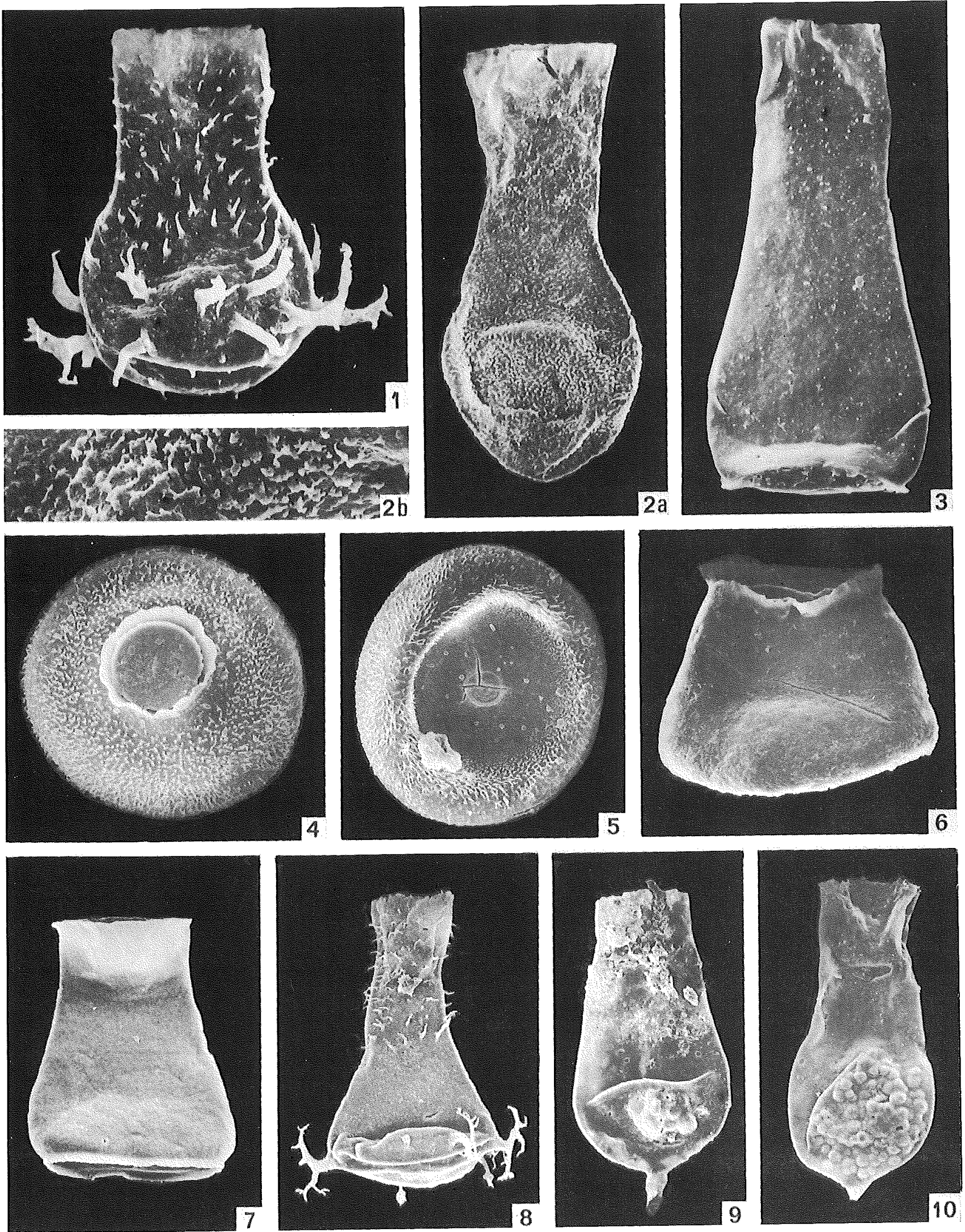


\section{PLANCHE 5}

\section{Chitinozoaires des sondages TRN 3 et EAL 1}

Fig. 1 - Gotlandochitina milanensis (Collinson \& Scott, 1958).

(x 300), sondage TRN 3, 1517 m, IGR 60305, (P. 38/4).

Fig. 2 - Ancyrochitina taouratinensis Boumendjel, 1985.

(x 350), sondage TRN 3, 1514 m, IGR 60308, (L. 35/4).

Fig. 3 et 4 - Linochitina jardinei Boumendjel, 1985.

3 - (x 400), sondage TRN 3, $1514 \mathrm{~m}$, IGR 60232, (O. 37/2).

4 - (x 400), sondage TRN 3, 1514 m, IGR 60312, (J. 35/3).

Fig. 5 - Bursachitina riclonensis (Paris, 1980).

(x 400), sondage TRN 3, $1587 \mathrm{~m}$, IGR 60231, (O. 41/4).

Fig. 6 a-b - Eisenackitina castor Jansonius, 1964, sensu Legault, 1973.

Sondage TRN 3, 1514 m, IGR 60232, (R. 40/1).

Fig. 7 a-b et 8 - Fungochitina fenestrata (Taugourdeau \& Jekhowsky, 1960).

Sondage TRN 3.

$7 \mathrm{a}-(x$ 350), $1327 \mathrm{~m}$, IGR 60325, (Q. 38).

$7 \mathrm{~b}$ - (x 1300), détail de la collerette ajourée du spécimen de la fig. 7a.

8 - (x 350), 1327,25 m, IGR 60323, (P. 39/3).

Fig. 9 - Fungochitina pilosa (Collinson \& Scott, 1958).

(x 400), sondage TRN 3, $1517 \mathrm{~m}$, IGR 60305, (Q. 38/4).

Tous les Chitinozoaires figurés sont conservés dans les collections de l'Institut de Géologie de Rennes (IGR). Le repéreur England-Finder a été utilisé pour définir les coordonnées des spores et des Chitinozoaires illustrés. 
Geobios

$\mathrm{n}^{0} \mathbf{2 1}$, fasc. 3
PI. 5

K. Boumendjel, S. Loboziak, F. Paris, P. Steemans \& M. Streel
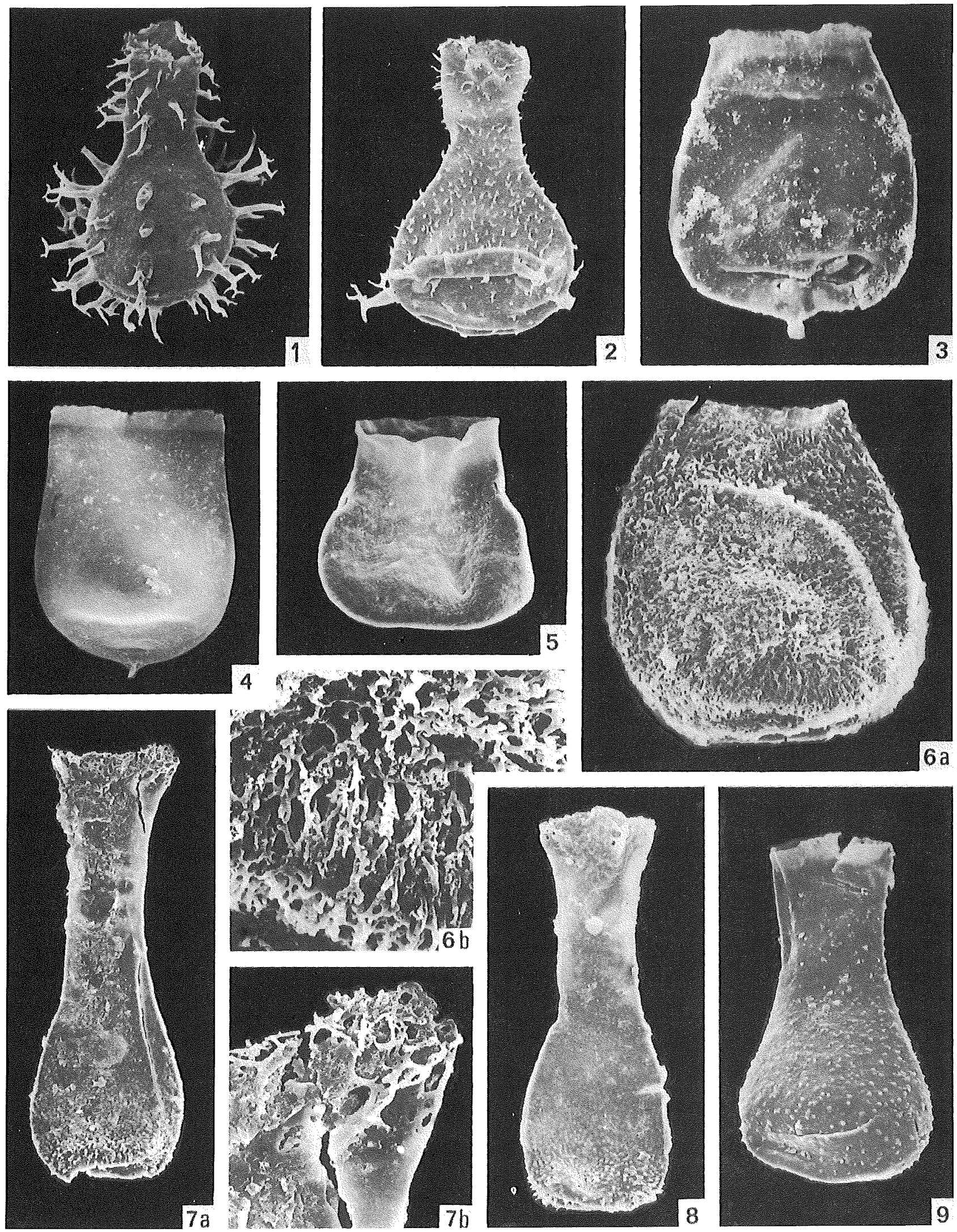